\title{
Patología dual en trastornos de ansiedad: recomendaciones en el tratamiento farmacológico
}

\section{Dual diagnosis in anxiety disorders: pharmacologic treatment recommendations}

\author{
Pilar Alejandra Sáiz Martínez*,*****, Luis Jimenez Treviño*****, Eva Ma Díaz Mesa**,***, \\ M a Paz García-Portilla González*******, Pedro Marina González*,***, Susana Al-Halabí******, \\ Néstor Szerman****,*****, Julio Bobes García*******, Pedro Ruiz****** \\ *Area de Psiquiatría. Universidad de Oviedo, Spain; **Centro de Investigación Biomédica en Red de Salud Mental, \\ CIBERSAM, Spain; ***Instituto de Investigación en Neurociencias del Principado de Asturias, INEUROPA, Spain; \\ ****Hospital Universitario Gregorio Marañón, Madrid, Spain; *****Presidente de la Sociedad Española de Patologia Dual, \\ Spain; ******Department of Psychiatry and Behavioural Sciences, University of Miami, Millar School of Medicine, USA.
}

\section{Resumen}

Los trastornos por uso de sustancias y los trastornos de ansiedad presentan una gran comorbilidad (entre el $18 \%$ y $37 \%$ ), lo cual complica el tratamiento y empeora el pronóstico de los pacientes (incluido mayor riesgo de suicidio). Son pocos los trabajos de investigación terapéutica que hayan abordado de forma específica el tratamiento farmacológico de los trastornos de ansiedad en caso de patología dual. La mayor parte de los autores apuestan por el abordaje de ambos trastornos de forma integral y simultánea. Los datos procedentes de la revisión de la literatura indican que los fármacos recomendados habitualmente en el tratamiento para los trastornos de ansiedad también son eficaces en el tratamiento de la ansiedad dual. Los antidepresivos ISRS constituyen el tratamiento de primera línea en los trastornos de ansiedad duales, mientras que las benzodiacepinas son fármacos a evitar. En los últimos años se observa una gran tendencia a utilizar fármacos antiepilépticos de última generación, los cuales muestran resultados prometedores en estudios abiertos y series de casos, en especial la pregabalina en el trastorno de ansiedad generalizada.

Palabras Clave: trastornos de ansiedad, patología dual, comorbilidad, tratamiento, recomendaciones.

\begin{abstract}
Anxiety disorders and substance use disorders are highly comorbid (between $18 \%$ and $37 \%$ ), and such comorbidity complicates treatment and worsens prognosis (including higher suicide risk). There are not many research works on the specific pharmacologic treatment of dual comorbid anxiety disorders. Most authors recommend a simultaneous approach of both, anxiety and substance use, disorders. Research data on pharmacotherapy suggest that psychotropics used in the treatment of anxiety disorders are also effective in dual diagnosis. SSRIs are considered first-line therapy in the treatment of dual anxiety while benzodiacepines should be avoided. New generation antiepileptic have shown efficacy in case series and open label studies in the latest years, thus being a promising treatment option for dual comorbid anxiety disorders, specially pregabalin in generalized anxiety disorder. Key Words: anxiety disorders, dual diagnosis, comorbidity, therapeutics, recommendations.
\end{abstract}


$\mathrm{L}$ os pacientes con trastornos de ansiedad (TA) son muy vulnerables a desarrollar otras patologías comórbidas. Tanto los grandes estudios epidemiológicos como estudios clínicos muestran una elevada asociación entre los distintos trastornos de ansiedad (TA) y los trastornos por uso de sustancias (TUS), que oscila entre el 18\% y el 37\% (Buckley y Brown, 2006; Fatseas, Denis, Lavie y Auriacombe, 2010; Grant et al., 2004; Kessler et al., 2006; Marmorstein, 2012; Merikangas et al., 1996; Regier et al., 1990). Esta asociación es especialmente llamativa cuando se consideran los trastornos relacionados con el uso de alcohol (Arias et al., 2013a) y trastornos de ansiedad específicos como la agorafobia o el trastorno de pánico (ver Tabla 1 donde se resumen los datos epidemiológicos de la asociación entre TA y TUS en diversos estudios realizados en población general). De igual modo ocurre con el cannabis y los trastornos de ansiedad generalizada o de pánico (Arias et al., 2013b). Cabe reseñar que las prevalencias de TA asociado a TUS, varían en función del ámbito de realización del estudio y de las sustancias incluidas en el mismo. En general, la asociación es menor en población general que en población clínica, ya que la presencia de un trastor- no mental facilita una mayor oportunidad de diagnóstico y tratamiento de la patología dual. No obstante, existen estudios que ponen de manifiesto que en muchas ocasiones existe infratratamiento de la patología comórbida (Terra et al., 2006).

La presencia de comorbilidad entre TA y TUS complica el tratamiento y empeora el pronóstico de ambos tipos de trastorno (Madoz, García, Luque, y Ochoa, 2013). Por ejemplo, se ha demostrado cómo la comorbilidad entre el uso de alcohol y el trastorno obsesivo compulsivo aumenta el riesgo de suicidio, o cómo los pacientes dependientes con trastornos de ansiedad no tratados tienen más posibilidad de recaídas (Ochoa, Salvador, Vicente, y Madoz, 2010; Pasche, 2012).

\section{Hipótesis explicativas de la relación entre TUS y TA}

De forma general, se consideran cuatro modos de asociación entre TUS y TA(Pasche, 2012):

1. el TA es primario y las sustancias se utilizan a modo de "automedicación" para paliar los síntomas de ansiedad: para poder confirmar la hipótesis de la automedicación, en primer lugar hay que establecer la secuencia temporal de la comorbilidad, de manera que

Tabla 1

Riesgo de patología dual (TUS y TA) en población general

\begin{tabular}{|c|c|c|c|c|c|c|c|c|}
\hline \multirow[t]{3}{*}{ Trastorno primario } & \multicolumn{2}{|c|}{$\begin{array}{c}\text { ECA } \\
\text { (Regier et al., 1990) }\end{array}$} & \multicolumn{2}{|c|}{$\begin{array}{c}\text { NESARC } \\
\text { (Conway et al., 2006; } \\
\text { Hasin et al., 2007) }\end{array}$} & \multirow{2}{*}{\multicolumn{2}{|c|}{$\begin{array}{c}\text { NCS-R } \\
\text { (Marmorstein, 2012) } \\
\text { OR* (IC 95\%) }\end{array}$}} & \multirow{2}{*}{\multicolumn{2}{|c|}{$\begin{array}{c}\text { ESEMeD } \\
\text { (Alonso et al., 2004) } \\
\text { OR* (IC 95\%) }\end{array}$}} \\
\hline & \multirow{2}{*}{$\begin{array}{l}\text { Preval- } \\
\text { vida }\end{array}$} & \multirow{2}{*}{$\begin{array}{l}\text { OR* } \\
\text { (IC 95\%) }\end{array}$} & \multirow{2}{*}{$\begin{array}{l}\text { Preval- } \\
\text { vida }\end{array}$} & \multirow{2}{*}{$\begin{array}{l}\text { OR* } \\
\text { (IC 95\%) }\end{array}$} & & & & \\
\hline & & & & & Abuso & Dependencia & Abuso & Dependencia \\
\hline TA y desarrollo de algún TUS & $23.7 \%$ & 1.7 & & & & & & \\
\hline Fobias & $22.9 \%$ & 1.6 & & & & & & \\
\hline TP & $35.8 \%$ & 2.9 & & & & & & \\
\hline TOC & $32.8 \%$ & 2.5 & & & & & & \\
\hline TUS (alcohol) y desarrollo de TA & $19.4 \%$ & 1.5 & & 2.3 & & & & \\
\hline Fobia & & 1.4 & & & & & & \\
\hline -Fobia social & & & & 2.3 & 3.03 & 4.01 & $2.7(1.5-4.7)$ & $2.7(0.6-12.3)$ \\
\hline -Fobia específica & & & & 2.2 & & & $2.0(1.3-3.2)$ & $3.3(1.3-8.4)$ \\
\hline TP & & 2.6 & & 2.4 & 3.71 & 4.93 & $3.3(1.8-5.8)$ & $6.8(2.2-21.1)$ \\
\hline -Con agorafobia & & & & 2.5 & & & & \\
\hline -Sin agorafobia & & & & 2.3 & & & & \\
\hline Agorafobia & & & & & 3.50 & 4.29 & $2.9(1.2-6.6)$ & $10.7(3.0-38.5)$ \\
\hline TAG & & & & 2.2 & 2.67 & 3.39 & $2.5(1.3-5.1)$ & $11.2(3.8-32.9)$ \\
\hline TOC & & 2.1 & & & & & & \\
\hline TEPT & & & & & & & $1.9(1.0-3.6)$ & $3.1(1.0-9.5)$ \\
\hline $\begin{array}{l}\text { TUS (otras sustancias) y } \\
\text { desarrollo de TA }\end{array}$ & $28.3 \%$ & $\begin{array}{l}2.5 \\
2.2\end{array}$ & $29.9 \%$ & 2.5 & & & & \\
\hline Fobia & & & $10.7 \%$ & 2.7 & 3.54 & 5.54 & & \\
\hline -Fobia social & & & $17.1 \%$ & 2.2 & & & & \\
\hline -Fobia específica & & 3.2 & & & 3.39 & 4.07 & & \\
\hline TP & & & $3.6 \%$ & 4.6 & & & & \\
\hline -Con agorafobia & & & $9.0 \%$ & 2.8 & & & & \\
\hline -Sin agorafobia & & & $9.2 \%$ & 3.3 & 2.84 & 3.11 & & \\
\hline TAG & & 3.7 & & & & & & \\
\hline TOC & & & & & & & & \\
\hline
\end{tabular}

*Riesgo de padecer trastorno comórbido del grupo expuesto frente al no expuesto

Nota. IC = Intervalo de confianza; $\mathrm{OR}=$ Odds ratio; TA = Trastorno de ansiedad; $\mathrm{TAG}=$ Trastorno de ansiedad generalizada; TEPT = Trastorno por estrés postraumático; TOC = Trastorno obsesivo-compulsivo; TP = Trastorno de pánico; TUS = Trastorno por uso de sustancias 
el TUS debería ser posterior al TA. Existen estudios a gran escala que han tratado de dilucidar la naturaleza de los patrones de comorbilidad entre los TA y los TUS. En concreto, el International Consortium in Psychiatric Epidemiology ha presentado datos en los que el inicio de los TA suele preceder al TUS (Marquenie et al., 2007; Merikangas et al., 1998).

2. el TUS es primario y los síntomas de ansiedad son consecuencia del uso o de la abstinencia de la sustancia: TAs como el trastorno de ansiedad generalizada o el trastorno por estrés postraumático, frecuentemente son secundarios al consumo de alcohol $\mathrm{u}$ otras sustancias. En estos casos, la pérdida de control sobre los consumos y/o la alteración de determinados sistemas de neurotransmisión facilitarían la aparición de determinados trastornos de ansiedad. En cambio, la agorafobia o el trastorno de pánico pueden aparecer de forma primaria o secundaria al TUS (Marmorstein, 2012; Sareen, Chartier, Paulus, y Stein, 2006). En este sentido, existen datos recientes que ponen de manifiesto que la dependencia de cannabis (Zvolensky et al., 2006), el consumo de más de un paquete diario de tabaco (Johnson et al., 2000), o el uso de alucinógenos (Bonn-Miller, Bernstein, Sachs-Ericsson, Schmidt y Zvolensky, 2007) se asocia con un riesgo incrementado de padecer, posteriormente, ataques de pánico.

3. el TA y el TUS no están relacionados pero interfieren entre sí, alterando la presentación y pronóstico mutuamente: en líneas generales, suele aceptarse que los trastornos fóbicos (sobre todo la fobia social) preceden al uso patológico de alcohol (Brady et al., 2005; Buckner et al., 2008; Marmorstein, 2012; Schneier et al., 2010; Terra et al., 2006) u otras sustancias (Buckner et al., 2008; Buckner y Schmidt, 2009; Marmorstein, 2012) por sus posibles efectos ansiolíticos, estando dicha comorbilidad asociada con una mayor gravedad del abuso o dependencia de la sustancia y con una menor tasa de búsqueda activa de tratamiento, más fracasos terapéuticos, mayores costes sanitarios por ingresos, consultas, y tiempo de tratamiento (Arias Horcajada, 2009; Scheneier et al., 2010).

4. el TA y el TUS son consecuencia de una base biológica o psicosocial común: los hallazgos actuales, en ocasiones contradictorios (Van Laar, van Dorsselaer, Monshouwer y de Graaf, 2007), sugieren que tanto el TUS como los TA pueden inducir el otro trastorno (Cosci, Schruers, Abrams y Griez, 2007). Así, por ejemplo, en el caso del alcohol se ha propuesto la existencia de un círculo vicioso en el que, a corto plazo, existe una disminución de la ansiedad inducida por el alcohol) que favorece su consumo, mientras que el consumo crónico y la abstinencia del mismo inducen síntomas de ansiedad, lo cual también refuerza los consumos (Kushner, Abrams y Borchardt, 2000), posiblemente en relación con mecanismos de hipersensibilidad al $\mathrm{CO}_{2}$ (Cosci et al., 2007). De igual modo, para complicar más aún el tema, hay que señalar la existencia de estudios que ponen de manifiesto la existencia de una agregación y transmisión familiar entre el trastorno por uso de alcohol y el trastorno de pánico (Goodwin et al., 2006; Cosci et al., 2007), lo cual indicaría que ambos trastornos comparten una causa común.

\section{Etiopatogenia de la ansiedad dual}

Estudios recientes ponen de manifiesto que el estrés podría ser un factor clave en la etiopatogenia del consumo de sustancias, así como de las recaídas, habiéndose demostrado cómo la exposición a situaciones estresantes en la infancia, o la presencia de estrés sostenido a lo largo de la vida, genera un mayor riesgo de comportamientos adictivos o aumenta la recompensa por consumo de ciertas sustancias (Cleck y Blendy, 2008).

Una de las hipótesis que explicaría esta relación desde un punto de vista neurobiológico la encontramos en el eje hipotálamo-hipófiso-adrenal (HPA). Durante los últimos años, diversos estudios han demostrado que la funcionalidad de este eje es modulada por el consumo de sustancias. El eje HPA se activa en roedores y primates tras la administración de la mayoría de sustancias de abuso produciendo un incremento de los niveles de ACTH y corticosterona plasmáticos (Kreek y Koob, 1998), que a su vez se correlacionan de modo positivo con conductas de autoadministración (Piazza et al., 1991). Por otra parte, la administración crónica de sustancias de abuso en animales da lugar a un incremento sostenido de la funcionalidad HPA, en el caso de los psicoestimulantes, o un descenso respecto a los efectos activadores iniciales de la droga, en el caso de morfina, nicotina o alcohol (Cleck y Blendy, 2008). De modo similar, los estudios realizados en humanos encuentran que la administración aguda de alcohol, cocaína, y nicotina se acompaña de un incremento de niveles de cortisol, mientras que la exposición aguda a opiáceos se acompaña de un descenso de los mismos. Por otra parte, la activación HPA persiste tras la adicción a cocaína, mientras que en el caso de adicción a opiáceos la respuesta HPA se va reduciendo a lo largo del tiempo (Cleck y Blendy, 2008).

Los datos obtenidos de estudios genéticos también apoyan esta relación, tras descubrirse cómo determinados polimorfismos implicados en la regulación del Eje HPA (Chong et al., 2006; Uhart, McCaul, Oswald, Choi y Wand, 2004; Wust et al., 2004) se asocian con la aparición de dependencia de alcohol (Oslin et al., 2003; Radel et al., 2005).

La influencia del eje HPA en las adicciones se ha explicado por la relación que existe entre la funcionalidad del eje HPA y el sistema dopaminérgico mesolímbico que, a su vez, podría contribuir a explicar la existencia de diferencias individuales en relación a la vulnerabilidad a la adicción (Uhart y Wand, 2009). El incremento de niveles séricos de 
glucocorticoides inducido por situaciones de estrés produce un incremento de la DA mesolímbica y a su vez facilita el consumo de psicoestimulantes y opiáceos en ratas (Piazza et al., 1990). Además, los niveles de cortisol tras la exposición a un estresor se correlacionan con los efectos subjetivos de las anfetaminas (Wand et al., 2007).

En resumen, un aumento de secreción de glucocorticoides o una mayor sensibilidad a los mismos, como sucedería en los pacientes con ansiedad, determinaría una mayor vulnerabilidad de la persona a desarrollar una dependencia a través de una potenciación de la actividad del sistema dopaminérgico mesolímbico (Manzanares et al., 2010) en los estadíos tempranos del consumo, mientras que el estrés crónico (que se acompaña de una situación de hipercortisolismo) se asocia a una disminución de la respuesta dopaminérgica mesolímbica, y crearía un estado de afecto negativo que facilitaría la continuidad de los consumos (Uhart y Wand, 2009).

Otro sistema de neurotransmisión que se ha relacionado con los trastornos de ansiedad es el endocannabinoide. La elevada prevalencia de TA entre consumidores habituales de cannabis ha dado lugar a diversas hipótesis que tratan de explicar dicha relación (ver Crippa et al., 2009). El uso de cannabis puede precipitar de modo agudo la ansiedad tanto por la acción directa de su principal principio psicoactivo, el delta-9-tetrahidrocannabinol, así como por la afectación cognitiva secundaria a la intoxicación aguda por cannabis.

\section{Protocolo de intervención}

\section{Evaluación diagnóstica}

Dada la frecuente asociación entre TA y TUS y que el diagnóstico y tratamiento precoz de dichas patologías mejora el pronóstico de ambas, es prioritario realizar un diagnóstico correcto e investigar la posibilidad de comorbilidad en pacientes que demandan tratamiento por problemas de ansiedad o TUS.

Una vez establecido el diagnóstico de comorbilidad sería necesario situar el diagnóstico de TA en una categoría específica (primario, efectos esperados o inducidos por sustancias). Para ello se recomienda tener presente las pautas de ayuda para el diagnóstico diferencial entre trastorno primario e inducido especificadas en la Tabla 9.

Uno de los métodos más reconocido para poder hacer esta distinción consiste en la observación del paciente durante un periodo de abstinencia, si bien no existe unanimidad en cuanto a la duración más apropiada del mismo. Se entiende que ante el consumo de sustancias de vida media larga como la metadona o ciertas BZD se requerirían tiempos de observación más prolongados que cuando se usan sustancias de vida media más corta como la cocaína. En general se recomienda 2-4 semanas de abstinencia con controles analíticos que aseguren que no hay consumo de sustancias. La persistencia de los síntomas de ansiedad después de este periodo, así como una historia familiar y/o personal de este tipo de trastornos y la constatación del inicio de la sintomatología ansiosa con antelación al uso de sustancias, serían datos a favor de un trastorno de ansiedad primario. Para una adecuada constatación de todo ello una evaluación clínica comprehensiva y que incluya una historia toxicológica exhaustiva es fundamental.

Por otra parte, hay que tener presente que para establecer un adecuado diagnóstico los instrumentos de evaluación psicométrica constituyen un elemento fundamental de ayuda (García-Portilla et al., 2011). Por ese motivo, en la Tabla 10 se resumen de modo simplificado una serie de instrumentos útiles tanto desde el punto de vista diagnóstico como para determinar la gravedad de las patologías existentes. Cabe reseñar que, la Asociación Americana de Psiquiatría, en la nueva versión de su Manual Diagnóstico y Estadístico de los Trastornos Mentales (DSM-5), recomienda diferentes instrumentos psicométricos para la facilitación de la evaluación diagnóstica y de la gravedad subyacente a los distintos trastornos. No obstante, dado que, en los trastornos que nos atañen, la mayoría de dichos instrumentos propuestos aún no han sido adaptados y validados al español, simplemente remitimos al lector interesado a la página web donde puede localizarlos (http://www.psychiatry.org/practice/dsm/ dsm5/ online-assessment-measures\#Disorder).

\section{Intervención terapéutica}

Uno de los aspectos claves del tratamiento de la ansiedad dual es el tratamiento específico del TUS. Por dicho motivo deben iniciarse las intervenciones farmacológicas y psicosociales necesarias para conseguir la abstinencia y el posterior mantenimiento de la misma.

No obstante, hay que tener presente que el tratamiento del TA es otro aspecto fundamental para mejorar el pronóstico de los pacientes y evitar el riesgo de recaídas en los consumos. En la Figura 1 se muestran, de modo resumido, los posibles pasos a seguir en el abordaje terapéutico de la ansiedad dual.

En caso de un TA inducido la propia abstinencia de las sustancias consumidas junto con el apoyo psicológico (y/o far-

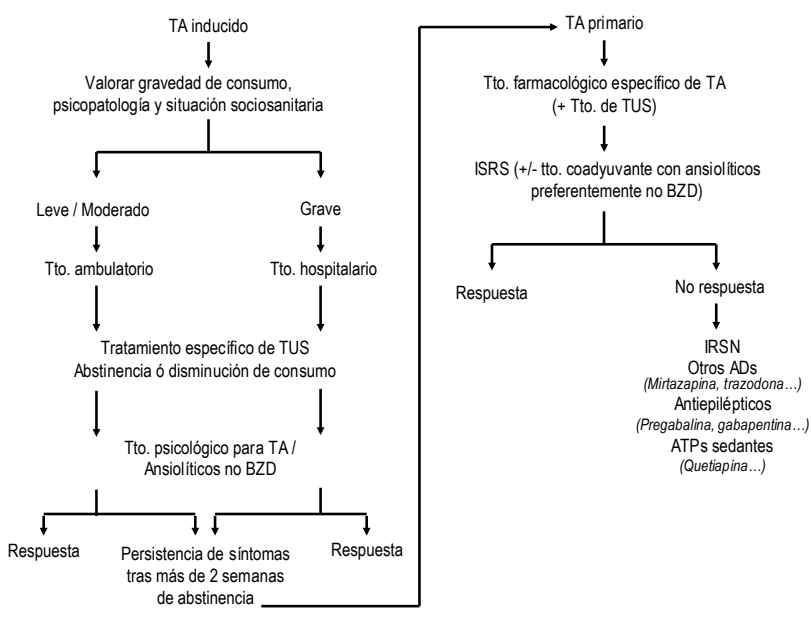

Figura 1. Algoritmo terapéutico de la ansiedad dual 
macológico) puede ser suficiente. En general, como ya se ha comentado con anterioridad, se recomienda el uso de ansiolíticos no BZD, siendo opciones adecuadas los antidepresivos de perfil sedativo (por ej., mirtazapina o trazodona), algunos antiepilépticos (pregabalina o gabapentina) o antipsicóticos atípicos de perfil sedativo a dosis bajas (por ej., la quetiapina).

En el caso de los TA primarios es necesario su abordaje terapéutico teniendo en cuenta las recomendaciones específicas realizadas para cada TA. No obstante, en líneas generales, cabe señalar que los ISRS a las dosis adecuadas para cada trastorno podrían ser una buena opción de inicio terapéutico, teniendo presente que en ocasiones puede ser necesario el tratamiento coadyuvante con otros fármacos ansiolíticos. En dicho caso la opción más segura sería la adición de fármacos no BZD. En el caso de falta de respuesta al ISRS (una vez que éste ha sido usado en dosis y tiempo adecuado), podría procederse al cambio por un IRSN (venlafaxina o duloxetina), otros antidepresivos de perfil sedativo (preferentemente mirtazapina o trazodona) o a la adición de otros fármacos de perfil ansiolítico como pregabalina o gabapentina o antipsicóticos de perfil sedativo. No obstante, hay que tener presente cual es el TA primario para, en función de el mismo, elegir las opciones terapéuticas más adecuadas.

\section{Abordaje terapéutico}

El abordaje terapéutico de los trastornos duales presenta importantes dificultades derivadas de las particulares características de estos trastornos, con tendencia a la recidiva y cronicidad, y de los patrones de conducta y relación interpersonal propios de los pacientes drogodependientes (Arias et al., 2013c).

Por lo tanto, el tratamiento ideal debería llevarse a cabo en un sistema asistencial integrado en el que se incluyan equipos multidisciplinares capaces de atender todas las necesidades terapéuticas del paciente a nivel psicopatológico, físico y social. Sin embargo, en la mayoría de los casos, la existencia de redes asistenciales diferentes y paralelas genera una gran dificultad para la atención de los pacientes con patología dual (Rubio, López-Muñoz y Álamo, 2002).

Los tratamientos utilizados en los pacientes duales suelen ser semejantes a los que utilizan cuando se presenta una sola patología y, en general, se considera que los tratamientos eficaces para tratar un determinado trastorno mental (distinto del TUS) también tienden a ser eficaces en los pacientes duales y viceversa (Ochoa et al., 2010).

\section{Recomendaciones farmacológicas generales para el manejo de la ansiedad dual}

En el momento actual carecemos de suficientes pruebas científicas de tipo I (ensayos clínicos controlados, metaanálisis o revisiones sistemáticas) o II (estudios controlados no aleatorizados bien diseñados) procedentes de estudios que hayan abordado de forma conjunta el tratamiento de los TA y TUS, de modo que la mayoría de aportaciones proce- den de las recomendaciones de expertos (Watkins, Hunter, Burnam, Pincus y Nicholson, 2005).

Los fármacos antidepresivos constituyen el tratamiento de primera línea en los TA (Ministerio de Sanidad y Consumo, 2008) y existen datos que ponen de manifiesto que el uso de determinados ISRS, como la paroxetina y la sertralina son eficaces en la reducción de la ansiedad en pacientes con dependencia de alcohol y TA comórbido, si bien los resultados sobre la dependencia de alcohol son dispares (Brady et al., 2005; Schadé et al., 2005).

Por otra parte, los antiepilépticos de nueva generación han demostrado eficacia tanto en el tratamiento de los diferentes TA como en el TUS. En las Tablas 2 a 7 se resumen los hallazgos en este sentido, y se puede apreciar cómo el TAG es el trastorno en el que se dispone de mejor nivel de pruebas científicas sobre la eficacia de los antiepilépticos, siendo la pregabalina el fármaco con más aval científico. En el caso de los TUS, las evidencias más notables se obtendrían, con la gabapentina, la pregabalina y el topiramato en la dependencia de alcohol, y con el topiramato en la dependencia a cocaína.

Las benzodiacepinas deben evitarse en este tipo de pacientes por el potencial desarrollo de tolerancia y dependencia al fármaco, como se ha destacado en diferentes estudios realizados en nuestro país (Secades et al., 2003; OECD, 2012), de manera que las guías clínicas desaconsejan su uso y, en caso de ser necesario, realizarlo sólo en tratamientos a corto plazo (Giner, 2005; NICE, 2011; San Molina, 2004).

En base a los datos existentes en la actualidad sobre el tratamiento de los TA con patología dual, se puede establecer una serie de recomendaciones terapéuticas generales que se recogen en forma de decálogo en la Tabla 8 .

\section{Tratamiento farmacológico de los trastornos de ansiedad específicos}

Trastorno de pánico. Existe cierta controversia acerca de si es necesario un tratamiento ansiolítico específico para los pacientes con abuso de sustancias primario, especialmente si se presentan crisis de angustia durante las primeras semanas de abstinencia. Sin embargo, si las crisis de angustia continúan en intensidad y frecuencia, estaría justificado un diagnóstico de trastorno de pánico (Gorman et al., 2006).

Muchos tipos de medicamentos han demostrado eficacia para el tratamiento del trastorno de pánico, como los ISRS, los ATC, IMAO y BZD. Sin embargo, no se dispone de estudios sistemáticos que evalúen la eficacia de estos tratamientos cuando el trastorno de pánico va asociado al abuso de sustancias. No obstante, los ISRS han mostrado un modesto efecto en la reducción del consumo de alcohol en algunos subgrupos de pacientes (Pettinati et al., 2000), no tienen potencial de abuso, son bien tolerados y relativamente seguros, por lo que son la elección más lógica en pacientes con este tipo de patología dual a las dosis habitualmente indicadas. 
Tabla 2

Antiepilépticos y trastorno de pánico (TP)

\begin{tabular}{|c|c|c|c|c|}
\hline Fármaco / estudio & $\begin{array}{l}\text { Tipo de estudio } \\
\text { (Nivel de } \\
\text { evidencia) }\end{array}$ & Dosis utilizada & Eficacia & Comentarios \\
\hline \multicolumn{5}{|l|}{ Gabapentina } \\
\hline Himmerich et al. (2007) & 1 caso clínico (III) & $300 \mathrm{mg} / \mathrm{día}$ & Eficaz y bien tolerado & $\begin{array}{l}\text { Dependencia a alprazolam e } \\
\text { intolerancia a carbamacepina }\end{array}$ \\
\hline Spila y Szumillo (2006) & 6 casos clínicos (III) & $300 \mathrm{mg} / \mathrm{día}$ & Eficaz y bien tolerado & Tto coadyuvante en TP \\
\hline Pande et al. (2000) & ECACP (I) & 600-3600mg/día & Eficaz y bien tolerado & Monoterapia en TP \\
\hline \multicolumn{5}{|l|}{ Tiagabina } \\
\hline Zwanzger et al. (2009) & Control placebo (II) & ------- & No diferencia entre grupos & Monoterapia en TP \\
\hline Sheehan et al. (2007) & Abierto (III) & 2-20mg/día & $\begin{array}{l}\text { Eficacia escasa y bien tolerado, } \\
\text { en general }\end{array}$ & Monoterapia en TP \pm agorafobia \\
\hline
\end{tabular}

Nota. ECACP = Ensayo clínico aleatorizado con placebo; TBP = Trastorno bipolar

Ahora bien, cuando se utilizan en este tipo de pacientes conviene acentuar las precauciones que se toman al principio del tratamiento para evitar el agravamiento de los síntomas de pánico debido al efecto activador de los ISRS, ya que en pacientes duales un empeoramiento de los síntomas de ansiedad podría incrementar el riesgo de recaídas en los consumo. La regla general de proceder a instaurar el tratamiento de modo progresivo, con un incremento paulatino desde dosis bajas, está especialmente indicado en estos pacientes ya que parecen presentar una sensibilidad mayor a este efecto y con ello un mayor riesgo de recaída.

Se debe prestar atención al periodo de latencia de inicio del efecto terapéutico de estos fármacos (2 a 6 semanas hasta alcanzar el efecto máximo) por el riesgo de recaídas en esta etapa (Brady y Verduin, 2005). En este tiempo se podría asociar una BZD, preferentemente de vida media larga (Rubio et al, 2002). Por ejemplo, el clonazepam ha sido utilizado con éxito en el tratamiento de crisis de angustia en pacientes consumidores de cocaína.

Existe cierta experiencia acerca de la eficacia de los ATC para el tratamiento del trastorno de pánico en pacientes bebedores de alcohol (Kranzler, 1996; Nunes, McGrath y Quitkin, 1995), pero han sido desplazados por los ISRS por la menor frecuencia y gravedad de los efectos secundarios.

De los inhibidores de recaptación de serotonina y noradrenalina (IRSN), la venlafaxina de liberación prolongada ha demostrado su eficacia en el tratamiento del trastorno de pánico con dosis entre 75 y $225 \mathrm{mg} /$ día (Bradwejn, Ahokas y Stein, 2005). Además, hay experiencia de su utilización en abusadores de alcohol con síntomas depresivos, observándose que es un fármaco bien tolerado, seguro, con escaso potencial de abuso e incluso con capacidad para mejorar los problemas relacionados con la dependencia del alcohol (García-Portilla et al., 2005).

A pesar de que las BZD han demostrado eficacia en el tratamiento del trastorno de pánico con o sin agorafobia, hay una tendencia a evitar su uso en pacientes con TUS y a usarlas con cautela en aquellos con antecedentes de este tipo de trastornos. No obstante, no existen datos publicados que avalen dicha práctica y se recomienda que la decisión de prescribir BZD en estos pacientes se tome en base a una valoración cuidadosa de las ventajas e inconvenientes de cada caso particular (Posternak y Mueller, 2001).

Aunque se ha sugerido la utilidad potencial de diversos fármacos antiepilépticos (carbamacepina, valproato, lamotrigina, gabapentina, pregabalina, tiagabina, topiramato) en el tratamiento del trastorno de pánico, sólo existen pruebas de nivel I en el caso de la gabapentina (ver Tabla 2). La principal ventaja de este tipo de fármacos, en especial los de más reciente introducción, radica en su buen perfil de seguridad, tolerabilidad y escaso potencial de abuso.

Trastorno de ansiedad generalizada (TAG). Los síntomas característicos del trastorno de ansiedad generalizada están presentes tanto en la intoxicación como en el síndrome de abstinencia de distintas sustancias (Chambless, Cherney y Caputo, 1987), lo cual complica de forma significativa el diagnóstico de TAG (Brady y Verduin, 2005), de manera que no debe realizarse hasta la completa remisión de la intoxicación y/o abstinencia (Back y Brady, 2008). En el caso de sustancias de vida media corta como la cocaína, el diagnóstico de TAG se puede hacer tras una semana de abstinencia, mientras que sustancias de vida media larga como la metadona o algunas benzodiacepinas requieren una demora de 4 a 8 semanas de abstinencia para poder realizar el diagnóstico de TAG (McKeehan y Martin, 2002).

En cuanto al tratamiento, numerosos fármacos han demostrado eficacia en el tratamiento del TAG, como los ISRS, IRSN, ATC, BZD, buspirona (retirada del mercado español) o antiepilépticos. Sin embargo, se recomienda evitar las BZD en el TAG en pacientes duales y, si se utilizan, debería de hacerse con mucha cautela respetando las indicaciones de la circular de 2000 de la Agencia Española del Medicamento, evitando superar las 8-12 semanas de tratamiento (Giner, 2005; San Molina, 2004). 
Tabla 3

Antiepilépticos y trastorno de ansiedad generalizada (TAG)

\begin{tabular}{|c|c|c|c|c|}
\hline Fármaco / estudio & $\begin{array}{l}\text { Tipo de estudio } \\
\text { (Nivel de } \\
\text { evidencia) }\end{array}$ & Dosis utilizada & Eficacia & Comentarios \\
\hline \multicolumn{5}{|l|}{ Pregabalina } \\
\hline Lydiard et al. (2010) & ECACP (I) & 150-600mg/día & $\begin{array}{l}\text { Eficaz en ansiedad (dosis } \nearrow \\
300 \mathrm{mg} / \text { día) y bien tolerado }\end{array}$ & $\begin{array}{l}\text { Monoterapia en TAG. Incluye } 6 \\
\text { ECACP previos }\end{array}$ \\
\hline Montgomery et al. (2009) & ECACP (I) & 150-600mg/día & $\begin{array}{l}\text { Eficaz en insomnio y ansiedad } \\
\text { (dosis } \nearrow 300 \mathrm{mg} / \text { día) }\end{array}$ & $\begin{array}{l}\text { Monoterapia en TAG. Incluye } 6 \\
\text { ECACP previos }\end{array}$ \\
\hline Stein et al. (2009) & ECACP (I) & 150-600mg/día & $\begin{array}{l}\text { Eficaz en síntomas } \mathrm{Gl} \text { y ansiedad } \\
\text { ldosis } \nearrow 300 \mathrm{mg} / \text { día }\end{array}$ & $\begin{array}{l}\text { Monoterapia en TAG. Incluye } 6 \\
\text { ECACP previos }\end{array}$ \\
\hline Stein et al. (2008) & ECACP (I) & 150-600mg/día & $\begin{array}{l}\text { Eficaz en síntomas depresivos } \\
\text { asociados a TAG }(300-450 \mathrm{mg} / \text { día) }\end{array}$ & $\begin{array}{l}\text { Monoterapia en TAG. Incluye } 6 \\
\text { ECACP previos }\end{array}$ \\
\hline Feltner et al. (2008) & ECACP (I) & 450mg/día & Eficaz en prevención de recaídas & Monoterapia en TAG \\
\hline \multicolumn{5}{|l|}{ Tiagabina } \\
\hline Pollack et al. (2008) & ECACP (I) & 4-16mg/día & No eficaz & $\begin{array}{l}\text { Monoterapia TAG. Incluye } 3 \\
\text { ECACP previos }\end{array}$ \\
\hline
\end{tabular}

Nota. ECACP = Ensayo clínico aleatorizado con placebo

Los ISRS y los IRSN son los fármacos más utilizados por la eficacia y seguridad en el TAG comórbido con TUS, si bien la trazodona también se ha utilizado con buenos resultados. Entre los nuevos antiepilépticos, la pregabalina (300-600 $\mathrm{mg}$ /día), es el único fármaco que acumula pruebas científicas de nivel I (ver Tabla 3). Su seguridad, tolerabilidad, escasas interacciones y bajo potencial de abuso, contribuyen a que este fármaco tenga las mejores perspectivas para el tratamiento del TAG en pacientes con TUS.

De igual modo, aunque con un nivel de evidencia más limitado, los antipsicóticos de segunda generación con per- fil sedativo (quetiapina, olanzapina y risperidona), a dosis bajas, podrían constituir otra alternativa para el tratamiento sintomático de la ansiedad (Gao, Sheehan y Calabresse, 2009; Sattar, Schultz, Arndt, Soundy y Petty, 2007).

\section{Trastorno por estrés postraumático (TEPT). El}

TEPT es uno de los trastornos de ansiedad más frecuentes en sujetos con TUS (Donovan et al., 2001). La presencia de TEPT incrementa entre dos y cuatro veces la posibilidad de abusar de sustancias (Back y Brady, 2008) del mismo modo que los consumidores de sustancias tienen un riesgo

Tabla 4

Antiepilépticos y trastorno por estrés postraumático (TEPT)

\begin{tabular}{|c|c|c|c|c|}
\hline Fármaco / estudio & $\begin{array}{l}\text { Tipo de estudio } \\
\text { (Nivel de } \\
\text { evidencia) }\end{array}$ & Dosis utilizada & Eficacia & Comentarios \\
\hline \multicolumn{5}{|l|}{ Valproato } \\
\hline Hamner et a.l (2009) & ECACP (I) & 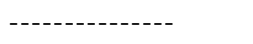 & No eficaz & Monoterapia en TEPT crónico \\
\hline Davis et al (2008) & ECACP (I) & Media = 2309mg/día & No eficaz & Monoterapia en TEPT crónico \\
\hline \multicolumn{5}{|l|}{ Gabapentina } \\
\hline Stein et al (2007) & ECACP (I) & 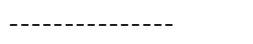 & No eficaz en prevención & Prevención de TEPT \\
\hline \multicolumn{5}{|l|}{ Oxcarbazepina } \\
\hline $\begin{array}{l}\text { Malek-Ahmadi y Hanretta } \\
\text { (2004) }\end{array}$ & 1 Caso clínico (III) & 1500mg/día & Eficaz en síntomas TEPT & TBP comórbido con TEPT \\
\hline \multicolumn{5}{|l|}{ Pregabalina } \\
\hline Pae et al (2009) & Abierto (III) & Dosis flexible & Eficaz y bien tolerado & Coadyuvante de ADT en TEPT \\
\hline \multicolumn{5}{|l|}{ Tiagabina } \\
\hline Davidson et al (2007) & ECACP (I) & 4-16mg/día & No eficaz, bien tolerado & Monoterapia en TEPT \\
\hline \multicolumn{5}{|l|}{ Topiramato } \\
\hline Alderman et al (2009) & Abierto (III) & $50-200 \mathrm{mg} / \mathrm{día}$ & $\begin{array}{l}\text { Mejoría sintomática. } \downarrow n^{0} \text { pacientes } \\
\text { con consumo excesivo de alcohol }\end{array}$ & Tto coadyuvante en TEPT \\
\hline Lindley et al (2007) & ECACP (I) & Dosis flexible & No eficaz, bien tolerado & Tto coadyuvante en TEPT \\
\hline Tucker et al (2007) & ECACP (I) & 400mg/día & $\downarrow$ algunos síntomas & Monoterapia en TEPT \\
\hline
\end{tabular}

Nota. ADT = Antidepresivos; ECACP = Ensayo clínico aleatorizado con placebo; TBP = Trastorno bipolar 
más elevado de TEPT, con prevalencias hasta del $50 \%$ en algunos estudios (North et al., 2002).

Además, la coexistencia de ambos trastornos dificulta notablemente el diagnóstico. Las manifestaciones clínicas del TEPT se pueden modificar por la intoxicación o la abstinencia de las distintas sustancias. Los pensamientos intrusivos pueden aumentar por la acción de sustancias estimulantes, alcohol, cannabis, y con la abstinencia de sedantes o estimulantes. El estado de alerta y activación se pueden reforzar con estimulantes o cannabis, o en los estados de abstinencia de éstas y también de alcohol, opiáceos o BZD. En general, la acción de sustancias estimulantes agrava los síntomas, mientras que las de acción depresora los alivian (Brady et al., 2005; Schäfer y Najavits 2007).

Los fármacos más utilizados en el tratamiento del TEPT por su eficacia, seguridad y tolerabilidad son los ISRS. Asimismo, se han obtenido buenos resultados con mirtazapina, trazodona, venlafaxina y bupropion, pero sin pruebas concluyentes de eficacia. También se ha observado cierta mejoría en los síntomas del TEPT con diversos antiepilépticos (ver Tabla 4), así como con antipsicóticos atípicos como la olanzapina, quetiapina y risperidona, especialmente útiles en casos graves con síntomas psicóticos (Ursano et al., 2006).

En los pocos estudios realizados para determinar los tratamientos más eficaces en pacientes con TEPT y TUS, los ISRS vuelven a ser los fármacos de elección por eficacia y escaso potencial de abuso (San Molina et al., 2005). Un estudio doble ciego con sertralina (150 mg/día) en pacientes con TEPT y alcoholismo puso de manifiesto la capacidad del fármaco para aliviar los síntomas del TEPT y para reducir el consumo de alcohol comparado con placebo (Brady et al., 2005). La venlafaxina podría utilizarse cuando predominan síntomas de ansiedad, y la mirtazapina o trazodona, dotados de un buen perfil sedativo, para el manejo del insomnio. En casos resistentes se han utilizado eutimizantes como valproato, así como los antip- sicóticos atípicos con perfil ansiolítico. No se recomiendan las BZD en pacientes con este trastorno dual (San Molina, 2004).

Existen estudios que han evaluado el efecto del tratamiento de sustitución con metadona en adictos a opiáceos con TEPT, y el del disulfiram y naltrexona en alcohólicos con dicho trastorno, apreciando que tales tratamientos eran eficaces para disminuir el uso de las sustancias problema pero sin efecto claro sobre los síntomas del TEPT (Schäfer y Najavits, 2007).

\section{Fobia social (trastorno de ansiedad social) (TAS).}

Las personas con TAS tienen unas altas tasas de comorbilidad con otros trastornos psiquiátricos, en especial con TUS (Brady et al., 2005). La elevada asociación entre TAS y consumo de alcohol (entre 2 y 3 veces mayor que la población general) puede explicarse con la hipótesis de la automedicación (Back y Brady, 2008). Según esta hipótesis el alcohol se utilizaría para disminuir la ansiedad en las interacciones sociales. En la mayoría de los casos el TAS es previo a los problemas por el uso de alcohol (Terra et al., 2006).

El diagnóstico precoz del TAS en usuarios de sustancias es de gran importancia porque el trastorno puede impedir una adecuada adherencia al tratamiento, especialmente a las actividades psicoterapéuticas. En estos casos no es necesario un largo periodo de observación en abstinencia pues el miedo a las interacciones sociales no es una característica específica de la intoxicación o del síndrome de abstinencia de sustancias, e incluso en los casos en los que aparece, como puede ocurrir en el contexto de consumo de cannabis o estimulantes, no cumple criterios de fobia social (Brady et al., 2005).

$\mathrm{Al}$ igual que en el resto de TA, apenas hay datos sobre la farmacoterapia del TAS cuando se asocia a uso de sustancias. No obstante, la paroxetina podría ser de utilidad en base a los resultados obtenidos en un estudio doble ciego aleatorizado y controlado con placebo, en pacientes con TAS y uso de alcohol, que puso de manifiesto una capacidad

Tabla 5

Antiepilépticos y fobia social

\begin{tabular}{|c|c|c|c|c|}
\hline Fármaco / estudio & $\begin{array}{l}\text { Tipo de estudio } \\
\text { (Nivel de } \\
\text { evidencia) }\end{array}$ & Dosis utilizada & Eficacia & Comentarios \\
\hline \multicolumn{5}{|l|}{ Valproato } \\
\hline Kinrys et al (2003) & Abierto (III) & 500-2500mg/día & Eficaz & Monoterapia en fobia social \\
\hline \multicolumn{5}{|l|}{ Gabapentina } \\
\hline Pande et al (1999) & ECACP (I) & 900-3600mg/día & Eficaz & Monoterapia en fobia social \\
\hline \multicolumn{5}{|l|}{ Pregabalina } \\
\hline Pande et al (2004) & ECACP (I) & 150 / 600mg/día & Eficaz $(600 \mathrm{mg} /$ día) y bien tolerado & Monoterapia en fobia social \\
\hline \multicolumn{5}{|l|}{ Tiagabina } \\
\hline Dunlop et al (2007) & Abierto (III) & 4-16mg/día & Eficaz y bien tolerado & Monoterapia en fobia social \\
\hline \multicolumn{5}{|l|}{ Topiramate } \\
\hline Van Ameringen et al (2004) & Abierto (III) & 400mg/día & Eficaz & Monoterapia en fobia social \\
\hline
\end{tabular}

Nota. ECACP = Ensayo clínico aleatorizado con placebo 
mayor que placebo para mejorar la ansiedad social, pero no se apreciaron cambios en la cantidad y frecuencia del consumo de alcohol. Sí se observó que en los pacientes sin paroxetina el consumo de alcohol estaba ligado a la intensidad de la ansiedad, mientras que en los pacientes tratados con paroxetina no se observaba esta relación (Thomas, Randall, Book y Randall, 2008). Otras opciones terapéuticas serían el resto de ISRS o la venlafaxina. Las BZD deben usarse con cautela, si bien pueden asociarse a los ISRS durante el periodo de latencia de inicio del efecto terapéutico (Brady et al., 2005).

Los nuevos antiepilépticos, especialmente la gabapentina y la pregabalina, podrían ser una excelente alternativa al uso de BZD, sobre todo en el caso del TAS asociado con trastorno por uso de alcohol (ver Tablas 5 y 7 ). Se ha publicado algún caso de eficacia de gabapentina en la reducción del craving y de la intensidad de la adicción en TUS con TAS comórbido (Verduin, McKay y Brady, 2007). A pesar de la utilidad de los IMAO en el TAS no se recomiendan cuando hay uso de alcohol u otras sustancias de abuso por las dificultades de manejo. En este caso, los inhibidores reversibles de la monoaminooxidasa (RIMA) son una alternativa si fracasan los tratamientos de primera línea.

Trastorno obsesivo-compulsivo (TOC). Existen cuantiosos datos acerca de la frecuente comorbilidad del TOC con otros trastornos mentales, sin embargo los datos de prevalencia de TOC y TUS, en población general, son más limitados que en otras patologías duales (Brady y Verduin, 2005). No obstante, cuando se presenta no suele plantear problemas diagnósticos porque los síntomas de TOC no se solapan con los de intoxicación o abstinencia de sustancias. Por ello, no suele ser necesario un periodo de observación prolongado en ausencia de consumo de sustancias para establecer el diagnóstico.

En cuanto al tratamiento farmacológico, no hay ensayos clínicos sobre el uso de psicofármacos en esta patología dual. Como en todos los casos de comorbilidad con uso de sustancias, se recomienda usar fármacos con escaso potencial de abuso, evitando, si es posible, el empleo de BZD. Los ISRS son las fármacos de primera elección a las dosis recomendadas para el TOC (Tiet y Mausbach, 2007), recordando que la acción terapéutica suele iniciarse entre la $6^{\mathrm{a}}$ y $8^{\mathrm{a}}$ semanas, y la eficacia máxima no se alcanza hasta la $12^{\mathrm{a}}$ semana. Si no hay respuesta se recomienda cambiar a otro ISRS; y en caso de que el cambio tampoco resulte efectivo, asociar o cambiar a clomipramina. En caso de asociar las dosis deben ser inferiores a las usadas en monoterapia. La clomipramina presenta mayor capacidad de disminuir el umbral convulsivo, así como de presentar interacciones con el alcohol, estimulantes y depresores del SNC (Brady y Verduin, 2005).

En casos resistentes podría ser de utilidad el tratamiento coadyuvante con fármacos antiepilépticos (ver Tabla 8) o con antipsicóticos atípicos de perfil más sedativo a dosis bajas. Estos fármacos podrían usarse en combinación con antidepresivos serotoninérgicos en pacientes en los que hayan fracasado otros tratamientos.

Tabla 6

Antiepilépticos y trastorno obsesivo compulsivo (TOC)

\begin{tabular}{|c|c|c|c|c|}
\hline Fármaco / estudio & $\begin{array}{l}\text { Tipo de estudio } \\
\text { (Nivel de } \\
\text { evidencia) }\end{array}$ & Dosis utilizada & Eficacia & Comentarios \\
\hline \multicolumn{5}{|l|}{ Carbamacepina } \\
\hline Aggarwal et al. (2009) & 1 caso clínico (III) & 1200mg/día & Mejoría + escitalopram (20mg/día) & TOC sin respuesta a ISRS \\
\hline Da Rocha et al. (2009) & 1 caso clínico (III) & 1200mg/día & Eficaz + clobazam (20mg/día) & TOC $2^{\circ}$ a epilepsia de lóbulo temporal \\
\hline \multicolumn{5}{|l|}{ Lamotrigina } \\
\hline Uzun (2010) & 1 caso clínico (III) & $150 \mathrm{mg} /$ día & Mejoría + clomipramina (225mg/día) & TOC resistente a clomipramina \\
\hline \multicolumn{5}{|l|}{ Pregabalina } \\
\hline Oulis et al. (2008a) & 1 caso clínico (III) & $450 \mathrm{mg} / \mathrm{día}$ & Mejoría & TOC inducido por BZD \\
\hline Oulis et al. (2008b) & 1 caso clínico (III) & 600mg/día & $\begin{array}{l}\text { Mejoría + sertralina ( } 400 \mathrm{mg} / \text { día) } \\
\text { + risperidona ( } 2 \mathrm{mg} / \text { día) }\end{array}$ & $\begin{array}{l}\text { TOC resistente a setralina + } \\
\text { risperidona }\end{array}$ \\
\hline \multicolumn{5}{|l|}{ Tiogabina } \\
\hline Oulis et al. (2009) & 1 caso clínico (III) & $15 \mathrm{mg} /$ día & $\begin{array}{l}\text { Mejoría + flovoxamina ( } 400 \mathrm{mg} / \\
\text { día) + risperidona (1 mg/día) }\end{array}$ & $\begin{array}{l}\text { TOC resistente a fluvoxamina + } \\
\text { risperidona }\end{array}$ \\
\hline \multicolumn{5}{|l|}{ Topiramate } \\
\hline Berlin et al. (en prensa) & ECACP (I) & $50-400 \mathrm{mg} / \mathrm{día}$ & Mejoría de compulsiones & $\begin{array}{l}\text { Tto coadyuvante en TOC } \\
\text { resistente a ISRS }\end{array}$ \\
\hline Hollander y Dell'Osso (2006) & 1 caso clínico (III) & $150 \mathrm{mg} /$ día & Mejoría + paroxetina (40mg/día) & TOC resistente a paroxetina \\
\hline $\begin{array}{l}\text { Van Ameringen et al. } \\
\text { (2006) }\end{array}$ & Serie de casos (III) & Media $=253 \mathrm{mg} / \mathrm{dí} a$ & Mejoría & $\begin{array}{l}\text { Tto coadyuvante en TOC } \\
\text { resistente a ISRS }\end{array}$ \\
\hline
\end{tabular}

Nota. ECACP = Ensayo clínico aleatorizado con placebo 
Tabla 7

Nuevos antiepilépticos y TUS*

\begin{tabular}{|c|c|c|c|c|}
\hline Fármaco / estudio & $\begin{array}{l}\text { Tipo de estudio } \\
\text { (Nivel de } \\
\text { evidencia) }\end{array}$ & Dosis utilizada & Eficacia & Comentarios \\
\hline \multicolumn{5}{|l|}{ Gabapentina } \\
\hline Brower et al. (2008) & ECACP (I) & $1500 \mathrm{mg} /$ día & Retrasa el inicio de ingesta excesiva & Dependencia alcohol + Insomnio \\
\hline Furieri et al. (2007) & ECACP (I) & $600 \mathrm{mg} / \mathrm{día}$ & $\downarrow$ consumo de alcohol y craving & Dependencia alcohol \\
\hline González et al. (2007) & ECACP (I) & 2400mg/día & No eficaz & $\begin{array}{l}\text { Dependencia cocaína }+ \\
\text { tratamiento con metadona }\end{array}$ \\
\hline Bisaga et al. (2006) & ECACP (I) & $3200 \mathrm{mg} /$ día & No eficaz & Dependencia cocaína \\
\hline Martínez-Raga et al. (2004) & Abierto (III) & 1800mg/día & Eficaz y bien tolerado & $\begin{array}{l}\text { Coadyuvante en sd abstinencia } \\
\text { heroína }\end{array}$ \\
\hline Bonnet et al. (2003) & ECACP (I) & 1600mg/día & No eficaz & Sd abstinencia alcohol \\
\hline \multicolumn{5}{|l|}{ Lamotrigina } \\
\hline Rubio et al. (2006) & Abierto (III) & $300 \mathrm{mg} / \mathrm{día}$ & $\downarrow$ CDT y craving & Dependencia alcohol + TBP \\
\hline Brown et al. (2006) & Abierto (III) & $300 \mathrm{mg} / \mathrm{dí} a$ & $\downarrow$ consumo y craving & Dependencia cocaína + TBP \\
\hline Berger et al. (2005) & $\begin{array}{l}\text { Abierto controlado } \\
\text { con placebo (II) }\end{array}$ & $\begin{array}{l}\text { Reserpina }=0.5 \mathrm{mg} / \text { día } \\
\text { Gabapentina }= \\
1800 \mathrm{mg} / \text { día }\end{array}$ & $\begin{array}{l}\text { Todas más eficaces que placebo } \\
\text { y bien toleradas }\end{array}$ & Dependencia cocaína \\
\hline Brown et al. (2003) & Abierto (III) & $\begin{array}{l}\text { Lamotrigina }=150 \\
\mathrm{mg} / \text { día }\end{array}$ & $\downarrow$ craving y bien tolerado & Dependencia cocaína + TBP \\
\hline Rosen et al. (1998) & Abierto (III) & $\begin{array}{l}\text { 300mg/día } \\
\text { 250-400mg/día }\end{array}$ & No eficaz y bien tolerado & $\begin{array}{l}\text { Sd abstinencia heroína } \\
\text { precipitado por naloxona }\end{array}$ \\
\hline \multicolumn{5}{|l|}{ Oxcarbacepina } \\
\hline Martinotti et al. (2007) & $\begin{array}{l}\text { Abierto aleatorizado } \\
\text { controlado con } \\
\text { naltrexona (II) }\end{array}$ & $\begin{array}{l}\text { OXC }=1500-1800 \mathrm{mg} / \text { día } \\
\text { OXC }=600-900 \mathrm{mg} / \text { día } \\
\mathrm{NTX}=50 \mathrm{mg} / \mathrm{día}\end{array}$ & Eficaz a dosis 1500-1800mg/día & $\begin{array}{l}\text { Prevención de recaídas en } \\
\text { dependencia alcohol }\end{array}$ \\
\hline Koethe et al. (2007) & ECACP (I) & - & No eficaz & Sd abstinencia alcohol \\
\hline Llopis y Castillo (2008) & Observacional (III) & 600-900mg/día & $\downarrow$ consumo, craving e impulsividad & Uso/dependencia cocaína \\
\hline Croissant et al. (2005) & Estudio de casos & 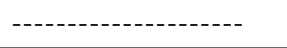 & Eficaz & Desintoxicación de BZD \\
\hline \multicolumn{5}{|l|}{ Pregabalina } \\
\hline Di Nicola et al. (2010) & Abierto (III) & 200-450mg/día & $\begin{array}{l}\downarrow \text { síntomas abstinencia y craving. } \\
\text { Bien tolerado }\end{array}$ & Desintoxicación alcohólica \\
\hline Martinotti et al. (2010a) & ECACP (I) & $150-450 \mathrm{mg} / \mathrm{dí}$ & Igual de eficaz que naltrexona & Dependencia alcohol \\
\hline Martinotti et al. (2010b) & ECACP (I) & $450 \mathrm{mg} / \mathrm{día}$ & Eficaz y bien tolerado & Sd abstinencia alcohol \\
\hline \multicolumn{5}{|l|}{ Tiagabina } \\
\hline Paparrigopoulos et al. (2009) & $\begin{array}{l}\text { Abierto aleatorizado } \\
\text { controlado (II) }\end{array}$ & & $\begin{array}{l}\text { Menor tasa de recaídas y bien } \\
\text { tolerado }\end{array}$ & $\begin{array}{l}\text { Coadyuvante de psicoterapia en } \\
\text { dependencia alcohol }\end{array}$ \\
\hline González et al. (2007) & ECACP (I) & $24 \mathrm{mg} / \mathrm{dí} a$ & $\begin{array}{l}\text { Eficaz en reducción consumo de } \\
\text { cocaína }\end{array}$ & $\begin{array}{l}\text { Dependencia cocaína }+ \\
\text { tratamiento con metadona }\end{array}$ \\
\hline Winhusen et al. (2007) & ECACP (I) & $20 \mathrm{mg} / \mathrm{dí} a$ & No eficaz & Dependencia cocaína \\
\hline Winhusen et al. (2005) & ECACP (I) & $20 \mathrm{mg} / \mathrm{dí} a$ & Mejoría de medidas subjetivas & Dependencia cocaína \\
\hline González et al. (2003) & ECACP (I) & $12-24 \mathrm{mg} / \mathrm{día}$ & $\begin{array}{l}\text { Eficaz (dosis } 24 \mathrm{mg} / \text { día) y bien } \\
\text { tolerado }\end{array}$ & $\begin{array}{l}\text { Dependencia cocaína + } \\
\text { tratamiento con metadona }\end{array}$ \\
\hline \multicolumn{5}{|l|}{ Topiramato } \\
\hline Florez et al. (2010) & Abierto (III) & $\begin{array}{l}\text { TPM }=200-400 \mathrm{mg} / \text { día } \\
\text { NTX }=50 \mathrm{mg} / \mathrm{día}\end{array}$ & Más eficaz que naltrexona & Dependencia alcohol \\
\hline Rubio et al. (2009) & ECACP (I) & - & Eficaz y bien tolerado & Dependencia alcohol \\
\hline Baltieri et al. (2009) & ECACP (I) & $300 \mathrm{mg} / \mathrm{dí} a$ & Eficaz en prevención de recaídas & Dependencia alcohol \\
\hline Flórez et al. (2008) & $\begin{array}{l}\text { Abierto controlado } \\
\text { con naltrexona (II) }\end{array}$ & $\begin{array}{l}\text { TPM }=200-400 \mathrm{mg} / \mathrm{día} \\
\mathrm{NTX}=50 \mathrm{mg} / \mathrm{dí} a\end{array}$ & Igual de eficaz que naltrexona & Dependencia alcohol \\
\hline Reis et al. (2008) & Abierto (III) & 25-300mg/día & $\downarrow$ craving y bien tolerado & Dependencia cocaína \\
\hline Bobes et al. (2004) & Abierto (III) & Máxima 400mg/día & $\downarrow$ consumo y craving & Dependencia cocaína / opiáceos \\
\hline Kampman et al. (2004) & ECACP (I) & $200 \mathrm{mg} /$ día & $\uparrow$ abstinencia & Dependencia cocaína \\
\hline Zullino et al. (2002) & Tres casos (III) & 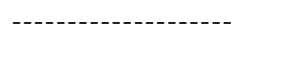 & Eficaz & $\begin{array}{l}\text { Coadyuvante en sd abstinencia } \\
\text { heroína }\end{array}$ \\
\hline
\end{tabular}

Nota. $C D T$ = Transferrina deficiente en carbohidratos; $E C A C P=$ Ensayo clínico aleatorizado con placebo; $T B P=$ Trastorno bipolar

*Sólo se incluyen los estudios con nivel más elevado de evidencia para cada grupo 
Tabla 8

Recomendaciones terapéuticas generales para el manejo de la ansiedad dual

1. El tratamiento ha de ser individualizado, teniendo presentes los recursos asistenciales disponibles en el entorno y las expectativas del paciente.

2. El tratamiento de ambos trastornos debería realizarse de modo simultáneo. No obstante, es importante establecer una adecuada secuenciación de los tratamientos debido a la frecuencia con la que la abstinencia se acompaña de síntomas de ansiedad y para evitar interacciones.

3. Se debe controlar la adherencia al tratamiento así como supervisar el posible mal uso o abuso de los psicofármacos pautados para manejar situaciones de estrés.

4. Se recomiendan apoyo psicosocial y entrenamiento en estrategias de afrontamiento para trastornos de ansiedad inducidos, reservándose el uso de medicación para ansiedad persistente y trastornos de ansiedad primarios.

5. Se debe maximizar el uso de los abordajes no-farmacológicos. La psicoterapia cognitivo-conductual ha demostrado eficacia en el abordaje de ambos tipos de trastorno, y un adecuado entrenamiento en relajación puede interrumpir el ciclo de la automedicación.

6. El tratamiento farmacológico de la ansiedad dual es, en general, semejante al de los TA no comórbidos, no estando contraindicado ningún psicofármaco. Al elegir la medicación se deben tener en cuenta las interacciones con la sustancia de abuso en caso de recaída, así como usar fármacos con el menor potencial de abuso.

7. Los ISRS son los fármacos de elección en el tratamiento de la ansiedad comórbida con el TUS, por el bajo potencial de abuso, escasas interacciones y relativa seguridad en caso de sobredosis. Las dosis son las mismas que la que se emplea en población sin TUS, cuidando las interacciones con otros fármacos habituales en pacientes abusadores de sustancias (metadona, antirretrovirales, aversivos del alcohol, etc.).

8. Los antidepresivos tricíclicos y tetracíclicos (ATC) deben utilizarse con precaución, dados los efectos secundarios e interacciones, así como el potencial para disminuir el umbral convulsivo, circunstancia a tener en cuenta en pacientes alcohólicos, con antecedentes de convulsiones o lesión cerebral. Debido a las interacciones con fármacos y alimentos, se desaconseja el empleo de los inhibidores de la monoaminooxidasa (IMAO).

9. Las benzodiacepinas (BZD) deben evitarse por el potencial de abuso (especialmente el alprazolam) y las peligrosas interacciones con alcohol, opiáceos y otros depresores del SNC. Están indicadas para el tratamiento de los cuadros de abstinencia y en las desintoxicaciones de alcohólicos o con dependencia a hipnosedantes. La Agencia Española del Medicamento (circular 3/2000) indica que la duración del tratamiento no debe de superar las 8-12 semanas, incluyendo el tiempo de retirada progresiva de las mismas.

10. 10. Los nuevos antiepilépticos podrían constituir un buen tratamiento alternativo al uso de BZD en el tratamiento de esta patología dual. De igual modo, aunque con evidencias más limitadas, los antipsicóticos con perfil sedativo a dosis bajas podrían constituir otra posible alternativa para el tratamiento sintomático.

Tabla 9

Pautas de ayuda para el diagnóstico diferencial entre trastorno primario ("independiente") e inducido

\begin{tabular}{ll}
\hline Trastorno primario / “independiente” & Trastorno inducido \\
\hline Edad de inicio de adicción posterior & Edad de inicio de adicción previo \\
Antecedentes personales psiquiátricos & No antecedentes personales de otros trastornos mentales \\
Antecedentes familiares psiquiátricos & Antecedentes familiares de adicciones \\
Persiste tras la abstinencia de sustancias & Remite con la abstinencia \\
Curso más recurrente & Curso menos recurrente (si no hay consumo) \\
Síntomas característicos del trastorno & Cuadros clínicos atípicos \\
Síntomas diferentes de lo esperable según tipo/cantidad de droga & Síntomas se corresponden con el perfil de la droga consumida \\
consumida & Mayor desestructuración familiar \\
Mejor estructura familiar & \\
\hline
\end{tabular}

Modificado de Arias Horcajada (2009) 
Pilar Alejandra Sáiz Martínez, Luis Jimenez Treviño, Eva Mª Díaz Mesa, Ma Paz García-Portilla González, Pedro Marina González, Néstor Szerman, Julio Bobes García, Pedro Ruiz

Tabla 10

Pautas guía para la evaluación psicométrica de la ansiedad dual en la práctica clínica cotidiana

\begin{tabular}{|c|c|c|c|}
\hline Area evaluada & Instrumento (autor) & Aspectos evaluados & Validación española \\
\hline $\begin{array}{l}\text { Orientación } \\
\text { diagnóstica }\end{array}$ & $\begin{array}{l}\text { MINI Entrevista Neuropsiquiátrica } \\
\text { Internacional (MINI) (Sheehan et al., 1997) } \\
\text { Entrevista de Investigación Psiquiátrica } \\
\text { para Trastornos Mentales y por Sustancias } \\
\text { (PRISM) (Hasin et al., 1996) }\end{array}$ & $\begin{array}{l}\text { Principales trastornos psiquiátricos del eje } \\
\text { I (criterios DSM-IV y CIE-10) } \\
\text { Comorbilidad psiquiátrica en pacientes con } \\
\text { consumo de alcohol y otras sustancias. } \\
\text { Discrimina trastornos mentales primarios, } \\
\text { inducidos por sustancias, efectos de } \\
\text { intoxicación y abstinencia. }\end{array}$ & $\begin{array}{l}\text { L. Ferrando, J. Bobes, J. } \\
\text { Gibert, M. Soto, O. Soto } \\
\text { Torrens et al. (2004) }\end{array}$ \\
\hline $\begin{array}{l}\text { Gravedad TA } \\
- \text { TP }\end{array}$ & $\begin{array}{l}\text { Escala de Pánico y Agorafobia de Bandelow } \\
\text { (PAS) (Bandelow, 1995) }\end{array}$ & Gravedad del trastorno de pánico & No existe \\
\hline - TAG & $\begin{array}{l}\text { Escala de Detección del TAG (Carroll y } \\
\text { Davidson, sin publicar) }\end{array}$ & $\begin{array}{l}\text { Identificación del trastorno de ansiedad } \\
\text { generalizada }\end{array}$ & Bobes et al. (2006) \\
\hline - TEPT & $\begin{array}{l}\text { Escala para el TEPT Administrada por el } \\
\text { Clínico (CAPS) (Blake et al., 1990) }\end{array}$ & $\begin{array}{l}\text { Presencia y gravedad de los síntomas del } \\
\text { trastorno por estrés postraumático }\end{array}$ & Bobes et al. (2000) \\
\hline - TAS & $\begin{array}{l}\text { Escala de Ansiedad Social de Liebowitz } \\
\text { (LSAS) (Liebowitz, 1987) }\end{array}$ & Gravedad de la fobia social & Bobes et al. (1999) \\
\hline - TOC & $\begin{array}{l}\text { Escala de Obsesiones y Compulsiones de } \\
\text { Yale-Brown (Y-BOCS) (Goodman et al., } \\
\text { 1989) }\end{array}$ & $\begin{array}{l}\text { Gravedad de los síntomas del trastorno } \\
\text { obsesivo compulsivo }\end{array}$ & Nicolini et al. (1996) \\
\hline $\begin{array}{l}\text { Gravedad TUS } \\
\text { - General }\end{array}$ & $\begin{array}{l}\text { Indice de Gravedad de la Adicción (versión } \\
\text { 6.0) (ASI6) (Alterman, sin publicar) }\end{array}$ & $\begin{array}{l}\text { Gravedad de la adicción entendida como } \\
\text { necesidad de tratamiento }\end{array}$ & Díaz Mesa et al. (2010) \\
\hline - Alcohol & $\begin{array}{l}\text { Test AUDIT (Saunders et al., 1993) } \\
\text { Escala para la Evaluación de la Abstinencia } \\
\text { Alcohólica (revisada) (CIWA-Ar) (Sullivan et } \\
\text { al., 1989) }\end{array}$ & $\begin{array}{l}\text { Detección de bebedores de riesgo } \\
\text { Gravedad del síndrome de abstinencia de } \\
\text { alcohol }\end{array}$ & $\begin{array}{l}\text { Rubio et al. (1998) } \\
\text { No existe }\end{array}$ \\
\hline - Opiáceos & $\begin{array}{l}\text { Escala Breve de Abstinencia a Opiáceos } \\
\text { (SOWS) (Gossop, 1990) }\end{array}$ & Gravedad de la abstinencia a opiáceos & No existe \\
\hline \multirow[t]{2}{*}{ - Cocaína } & $\begin{array}{l}\text { Cuestionario de Craving de Cocaína (CCQ) } \\
\text { (Tiffany et al., 1993) }\end{array}$ & Intensidad del craving de cocaína & Tejero et al. (2003a) \\
\hline & $\begin{array}{l}\text { Escala de Valoración de la Gravedad } \\
\text { Selectiva para Cocaína (CSSA) (Kampman } \\
\text { et al., 1998) }\end{array}$ & $\begin{array}{l}\text { Sintomatología inicial de la abstinencia a } \\
\text { cocaína }\end{array}$ & Tejero et al. (2003b) \\
\hline - BZD & $\begin{array}{l}\text { Cuestionario de Síntomas de Retirada de } \\
\text { BZD (BWSQ) (Tyrer et al., 1990) }\end{array}$ & $\begin{array}{l}\text { Gravedad del síndrome de retirada de } \\
\text { benzodiacepinas }\end{array}$ & No existe \\
\hline \multirow[t]{3}{*}{ Suicidabilidad } & $\begin{array}{l}\text { Escala SAD PERSONS (Patterson et al., } \\
\text { 1983) }\end{array}$ & $\begin{array}{l}\text { Riesgo de comportamiento suicida en base } \\
\text { a factores clínicos y sociodemográficos }\end{array}$ & No existe \\
\hline & $\begin{array}{l}\text { Escala Columbia para Evaluar el Riesgo de } \\
\text { Suicidio (Posner et al., 2011) }\end{array}$ & $\begin{array}{l}\text { Evaluación conjunta de pensamientos } \\
\text { suicidas (ideación suicida) y de } \\
\text { comportamientos suicidas }\end{array}$ & En fase de realización \\
\hline & $\begin{array}{l}\text { Escala de Intencionalidad Suicida de Beck } \\
\text { (SIS) (Beck et al., 1974) }\end{array}$ & $\begin{array}{l}\text { Características de la tentativa suicida } \\
\text { realizada }\end{array}$ & No existe \\
\hline
\end{tabular}

Modificado de García-Portilla et al (2011) y Sáiz et al (2011)

Nota. BZD = Benzodiacepinas; TA = Trastorno de ansiedad; TAG = Trastorno de ansiedad generalizada; TAS = Fobia social; TEPT = Trastorno por estrés postraumático; TOC = Trastorno obsesivo-compulsivo; TP = Trastorno de pánico; TUS = Trastorno por uso de sustancias 


\section{Tratamiento psicológico de la ansiedad dual}

Si bien el abordaje psicológico de los TA duales no es objeto de revisión en el presente trabajo, nunca se debe olvidar la necesidad de hacer un enfoque integral bio-psicosocial en el abordaje de este tipo de trastornos.

En este sentido, merece la pena hacer una breve referencia a la psicoterapia cognitivo-conductual, dada su eficacia demostrada en el tratamiento de los TA (Baker, Thornton, Hiles, Hiles y Lubman, 2012; Hesse, 2009).

$\mathrm{Al}$ igual que ocurre con los tratamientos farmacológiocos, apenas existen estudios sobre el tratamiento psicológico de los TA con TUS comórbidos (Baker et al., 2012), y los datos de estos trabajos no son concluyentes (Hobbs, Kushner, Lee, Reardon y Maurer, 2011). La psicoterapia cognitivo-conductual unida a la entrevista motivacional, han resultado eficaces en el tratamiento del abuso de alcohol en pacientes con ansiedad comórbida, especialmente cuando se realizan programas de intervención prolongados (Baker et al., 2012), si bien existen estudios en los que no se demuestra que el tratamiento psicológico simultáneo de TA y TUS aporte ventajas (Hesse, 2009; Randall, Thomas y Thevos, 2001). De hecho, alguno de estos trabajos recomienda que la intervención psicológica se haga de forma secuencial o escalonada (Baker et al., 2012).

\section{Limitaciones de la revisión}

Para la elaboración del presente trabajo, se ha realizado una búsqueda bibliográfica en Medline, revisando más de 70 estudios con diferente metodología. La variedad de estudios, así como la participación de cada autor en partes específicas del trabajo, ha imposibilitado la inclusión en las tablas de las medidas eficacia y de significación estadística de los estudios referenciados. Para acceder a esta información con mayor detalle, se remite al lector a las diferentes referencias bibliográficas.

\section{Conflicto de intereses}

No existen conflictos de intereses en la elaboración de este artículo de revisión. Los autores no han recibido financiación para la elaboración de este trabajo.

\section{Referencias}

Aggarwal, A., Jain, M., Garg, A., y Jiloham R. C. (2009). Carbamazepine for serotonin reuptake inhibitor nonresponder case of obsessive compulsive disorder. Indian Journal of Medical Sciences, 63, 468-469. doi: 10.4103/0019-5359.57644

Alamo, C., López-Muñoz, F., y Cuenca, E. (2002) Bases neurobiológicas del trastorno dual: hipótesis etiopatogénicas. En: G. Rubio, F. López-Muñoz C. Alamo, J.
Santo-Domingo (Eds.), Trastornos psiquiátricos y abuso de sustancias (pp.107-131). Madrid: Editorial Médica Panamericana.

Alderman, C. P., McCarthy, L. C., Condon, J. T., Marwood, A. C., y Fuller, J. R. (2009). Topiramate in combat-related posttraumatic stress disorder. The Annals of Pharmacotherapy, 43, 635-641.

Alonso, J., Angermeyer, M. C., Bernertm S., Bruffaerts, R., Brugha, T. S., Bryson, H.,... ESEMeD/MHEDEA 2000 Investigators, European Study of the Epidemiology of Mental Disorders (ESEMeD) Project. (2004). 12-Month comorbidity patterns and associated factors in Europe: results from the European Study of the Epidemiology of Mental Disorders (ESEMeD) project. Acta Psychiatrica Scandinavica, 420, 28-37.

Arias Horcajada, F. (2009). Prevalencia. En: J. Bobes García, y M. Casas Brugué (Coord.), Manejo clínico del paciente con patología dual (pp. 25-40). Barcelona: Socidrogalcohol.

Arias, F., Szerman, N., Vega, P., Mesias, B., Basurte, I., Morant, C.,... Babin, F. (2013a). Alcohol abuse or dependence and other psychiatric disorders. Madrid study on the prevalence of dual pathology. Mental Health and Substance Use, 6, 339-350. DOI:10.1080/17523281.2012.748674

Arias, F., Szerman, N., Vega, P., Mesias, B., Basurte, I., Morant, C.,... Babin,F. (2013b). Abuso o dependencia al cannabis y otros trastornos psiquiátricos. Estudio Madrid sobre prevalencia de patología dual. Actas Españolas de Psiquiatría, 41, 123-130.

Arias, F., Szerman, N., Vega, P., Mesias, B., Basurte, I., Morant, C.,... Babín, F. (2013c). Estudio Madrid sobre prevalencia y características de los pacientes con patología dual en tratamiento en las redes de salud mental y de atención al drogodependiente. Adicciones, 25, 118-127.

Armeli, S., Conner, T. S., Covault, J., Tennen, y H., Kranzler, H. R. (2008). A serotonin transporter gene polymorphism (5-HTTLPR), drinking-to-cope motivation, and negative life events among college students. Journal of Studies on Alcohol and Drugs, 69, 814-823.

Back, S. E., y Brady, K. T. (2008). Anxiety disorders with comorbid substance use disorders: diagnostic and treatment considerations. Psychiatric Annals, 38, 724-729

Baker, A. L., Thornton, L. K., Hiles, S., Hiles, y L., Lubman, D. I. (2012) Psychological interventions for alcohol misuse among people with co-occurring depression or anxiety disorders: a systematic review. Journal of Affective Disorders, 137, 217-229 doi: 10.1016/j.jad.2011.08.004

Baltieri, D. A., Daró, F. R., Ribeiro, P. L., y de Andrade, A. G. (2008). Comparing topiramate with naltrexone in the treatment of alcohol dependence. Addiction, 103, 20352044.

Bandelow, B. (1995). Assessing the efficacy of treatments for panic disorder and agoraphobia. II. The Panic and Agoraphobia Scale. International Clinical Psychopharmacology, 10, 73-81. 
Bartels, M., Van den Berg, M., Sluyter, F., Boomsma, D. I., y de Geus, E.J. (2003) Heritability of cortisol levels: review and simultaneous analysis of twin studies. Psychoneuroendocrinology, 28, 121-137.

Beck, A. T., Schuyler, D., y Herman, I. (1974). Development of suicidal intent scales. En: A. T. Beck, H. L. P. Resnick, D. J. Lettieri (Eds.), The prediction of suicide. (pp 45-46). Bowie: Charles Press.

Berger, S. P., Winhusen, T. M., Somoza, E. C., Harrer, J. M., Mezinskis, J. P., Leiderman, D. B.,... Elkashef, A. (2005). A medication screening trial evaluation of reserpine, gabapentin and lamotrigine pharmacotherapy of cocaine dependence. Addiction, 100, 58-67.

Berlin, H. A., Koran, L. M., Jenike, M. A., Shapira, N. A., Chaplin W., Pallanti, S., y Hollander, E. (2011). Double-blind, placebo-controlled trial of topiramate augmentation in treatment-resistant obsessive-compulsive disorder. Journal of Clinical Psychiatry, 72, 716-721

Bisaga, A., Aharonovich, E., Garawi, F., Levin, F. R., Rubin, E., Raby, W. N., y Nunes, E. V. (2006). A randomized placebo-controlled trial of gabapentin for cocaine dependence. Drug and Alcohol Dependence, 81, 267-274.

Blake, D. D., Weathers, F. W., Nagy, L. M., Kaloupek, D. G., Klauminzer, G., Charney, D. S., y Krane, T. M. (1990). A clinician rating scale for assessing current and lifetime PTSD: the CAPS-1. Behavior Therapist, 13, 187-188.

Bobes, J., Badía, X., Luque, A., García, M., González, M. P., y Dal-Ré, R. (1999) Validation of the Spanish version of the Liebowitz social anxiety scale, social anxiety and distress scale and Sheehan disability inventory for the evaluation of social phobia. Medicina Clinica (Barcelona), 112, 530-538.

Bobes, J., Calcedo-Barba, A., García, M., François, M., Rico-Villademoros, F., González, M. P,... Grupo Español de Trabajo para el Estudio del Trastorno por Estrés Postraumático. (2000). Evaluation of the psychometric properties of the Spanish version of 5 questionnaires for the evaluation of post-traumatic stress syndrome. Actas Españolas de Psiquiatria, 28, 207-218.

Bobes, J., Carreño, J. E., Gutiérrez, C. E., San Narciso, G. I., Antuña, M. J., Díaz, T.,... García-García, M. (2004). Study of effectiveness of craving control with topiramate in patients with substance dependence disorders. Actas Españolas de Psiquiatria, 32, 299-306.

Bobes. J., García-Calvo, C., Prieto, R., García-García, M., y Rico-Villademoros, F., Grupo Español de Trabajo para la validación de la versión española de la Escala de Detección del Trastorno de Ansiedad Generalizada según DSM-IV (Escala de TAG de Carroll y Davidson). (2006). Psychometric properties of the Spanish version of the screening scale for DSM-IV Generalized Anxiety Disorder of Carroll and Davidson. Actas Españolas de Psiquiatria, 34, 83-93.

Bonnet, U., Banger, M., Leweke, F. M., Specka, M., Müller, B. W., Hashemi, T.,... Gastpar, M. (2003). Treatment of acute alcohol withdrawal with gabapentin: results from a controlled two-center trial. Journal of Clinical Psychopharmacology, 23, 514-519.

Bonn-Miller, M. O., Bernstein, A., Sachs-Ericsson, N., Schmidt, N. B., y Zvolensky, M. J. (2007). Associations between psychedelic use, abuse, and dependence and lifetime panic attack history in a representative sample. Journal of Anxiety Disorders, 21, 730-741.

Bradwejn, J., Ahokas, A., y Stein, D. J. (2005). Venlafaxine extended-realease capsules in panic disorder: flexible-dose, double-blind, placebo-controlled study. British Journal of Psychiatry, 187, 352-359.

Brady, K. T., y Verduin, M. L. (2005). Pharmacotherapy of comorbid mood, anxiety, and substance use disorders. Substance Use E Misuse, 40, 2021-2041

Brady, K. T., Sonnem S., Antonm R. F., Randallm C. L., Back, S. E., y Simpson, K. (2005). Sertraline in the treatment of co-occurring alcohol dependence and posttraumatic stress disorder. Alcoholism: Clinical and Experimental Research, 29, 395-401.

Brower, K. J., Myra Kim, H., Strobbe, S., Karam-Hage, M. A., Consens, y F., Zucker, R. A. (2008). A randomized double-blind pilot trial of gabapentin versus placebo to treat alcohol dependence and comorbid insomnia. Alcoholism: Clinical and Experimental Research, 32, 1429-1438.

Brown, E. S., Nejtek, V. A., Perantie, D. C., Orsulak, P. J., y Bobadilla, L. (2003) Lamotrigine in patients with bipolar disorder and cocaine dependence. Journal of Clinical Psychiatry, 64, 197-201.

Brown, E. S., Perantie, D. C., Dhanani, N., Beard, L., Orsulak, P., y Rush, A.J. (2006) Lamotrigine for bipolar disorder and comorbid cocaine dependence: a replication and extension study. Journal of Affective Disorders, 93, 219-222.

Buckley, P. F., y Brown, E. S. (2006). Prevalence and consequences of dual diagnosis. Journal of Clinical Psychiatry, $67, \mathrm{e} 01$.

Buckner, J. D., Schmidt, N. B., Lang, A. R., Small, J. W., Schlauch, R. C., y Lewinsohn, P. M. (2008) Specificity of social anxiety disorder as a risk factor for alcohol and cannabis dependence. Journal of Psychiatry Research, 42, 230-239.

Buckner, J. D., y Schmidt, N. B. (2009). Social anxiety disorder and marijuana use problems: the mediating role of marijuana effect expectancies. Depression Anxiety, 26, 864-870.

Caspi, A., Sugden, K., Moffitt, T. E., Taylor, A., Craig, I. W., Harrington, H.,... Poulton, R. (2003). Influence of life stress on depression: moderation by a polymorphism in the 5-HTTLPR gene. Science, 301, 386-389.

Chambless, D. L., Cherney, J., y Caputo, G. C. (1987) Anxiety disorders and alcoholism: a study with inpatient alcoholics. Journal of Anxiety Disorders, 18, 29-40.

Chong, R. Y., Oswald, L., Yang, X., Uhart, M., Lin, P. I., y Wand, G. S. (2006) The mu-opioid receptor polymor- 
phism A118G predicts cortisol responses to naloxone and stress. Neuropsychopharmacology, 31, 204-211.

Cleck, J. N., y Blendy, J. A. (2008) Making a bad thing worse: adverse effects of stress on drug addiction. The Journal of Clinical Investigation, 118, 454-461.

Conway, K. P., Compton, W., Stinson, F. S., y Grant, B. F. (2006). Lifetime comorbidity of DSM-IV mood and anxiety disorders and specific drug use disorders: Results from the National Epidemiologic Survey on Alcohol and Related Conditions. Journal of Clinical Psychiatry, 67, 247-257.

Cosci, F., Schruers, K. R., Abrams, K., y Griez, E. J. (2007). Alcohol use disorders and panic disorder: a review of the evidence of a direct relationship. Journal of Clinical Psychiatry, 68, 874-880.

Covault, J., Tennen, H., Armeli, S., Conner, T. S., Herman, A. I., Cillessen, A. H., y Kranzler, H. R. (2007) Interactive effects on the serotonin transporter 5-HTTLPR polymorphism and stressful life events on college student drinking and drug use. Biological Psychiatry, 61, 609-616.

Crippa, J.A., Zuardi, A.W., Martín-Santos, R., Bhattacharyya, S., Atakan, Z., McGuire, P., y Fusar-Poli, P. (2009). Cannabis ans anxiety: a critical review of the evidence. Human Psychopharmacology: Clinical and Experimental, 24, 515-523.

Croissant, B., Grosshans, M., Klein, O., Dile, A., y Mann, K. (2005). Scheme-based benzodiazepine detoxification with oxcarbazepine - a case report. Pharmacopsychiatry, 38, 222-223.

Da Rocha, F. F., Bamberg, T. O., Lage, N. V., Correa, H., y Teixeira, A. L. (2009). Obsessive-compulsive disorder secondary to temporal lobe epilepsy with response to carbamazepine treatment. Progress in Neuropsychopharmacoly and Biological Psychiatry, 33, 568-569.

Davidson, J. R., Brady, K., Mellman, T. A., Stein, M. B., y Pollack, M. H. (2007). The efficacy and tolerability of tiagabine in adult patients with post-traumatic stress disorder. Journal of Clinical Psychopharmacology, 27, 85-88.

Di Chiara, G., Bassareo, V., Fenu, S., De Luca, M. A., Spina, L., Cadoni, C.,... Lecca, D. (2004). Dopamine and drug addiction: the nucleus accumbens shell connection. Neuropharmacology, 47, 227-241.

Di Nicola, M., Martinotti, G., Tedeschi, D., Frustaci, A., Mazza, M., Sarchiapone, M.,... Janiri, L. (2010). Pregabalin in outpatient detoxification of subjects with mild-to-moderate alcohol withdrawal syndrome. Human Psychopharmacology, 25, 268-275.

Díaz Mesa, E. M., García-Portilla, P., Sáiz, P. A., Bobes-Bascarán, T., Casares, M. J., Fonseca, E.,... Bobes, J. (2010). Psychometric performance of the 6th version of the Addiction Severity Index in Spanish (ASI-6). Psicothema, 22, 513-519.

Donovan, B., Padin-Rivera, E., y Kowaliw, S. (2001). "Transcend": initial outcomes from a posttraumatic stress disorder/substance abuse treatment program. Journal of Traumatic Stress, 14, 757-772.
Dunlop, B. W., Papp, L., Garlow, S. J., Weiss, P. S., Knight, B. T., y Ninan, P. T. (2007). Tiagabine for social anxiety disorder. Human Psychopharmacology, 22, 241-244.

Fatseas, M., Denis, C., Lavie, E., y Auriacombe, M. (2010). Relationship between anxiety disorders and opiate dependence-a systematic review of the literature: Implications for diagnosis and treatment. Journal of Substance Abuse Treatment, 38, 220-230.

Federenko, I. S., Nagamine, M., Hellhammer, D. H., Wadhwa, P. D., y Wüst, S. (2004). The heritability of hypothalamus pituitary adrenal axis responses to psychosocial stress is context dependent. The Journal of Clinincal Endocrinology and Metabolism, 89, 6244-6250.

Feinn, R., Nellissery, M., y Kranzler, H. R. (2005). Meta-analysis of the association of a functional serotonin transporter promoter polymorphism with alcohol dependence. American Journal of Medical Genetics, 133B, 79-84.

Feltner, D., Wittchen, H. U., Kavoussi, R., Brock, J., Baldinetti, F., y Pande, A. C. (2008). Long-term efficacy of pregabalin in generalized anxiety disorder. International Clinical Psychopharmacology, 23, 18-28.

Flórez, G., García-Portilla, P., Alvarez, S., Saiz, P. A., Nogueiras, L., y Bobes, J. (2008). Using topiramate or naltrexone for the treatment of alcohol-dependent patients. $\mathrm{Al}$ coholism: Clinical and Experimental Research, 32, 1251-1259.

Flórez, G., Saiz, P. A., García-Portilla, P., Alvarez, S., Nogueiras, L., y Bobes, J. (2010). Topiramate for the treatment of alcohol dependence: comparison with naltrexone. European Addiction Research, 17, 29-36.

Furieri, F. A., y Nakamura-Palacios, E. M. (2007). Gabapentin reduces alcohol consumption and craving: a randomized, double-blind, placebo-controlled trial. Journal of Clinical Psychiatry, 68, 1691-1700.

Gambarana, C., Masi, F., Tagliamonte, A., Scheggi, S., Ghiglieri, O., y De Montis, M. G. (1999). A chronic stress that impairs reactivity in rats also decreases dopaminergic transmission in the nucleus accumbens: a microdialysis study. Journal of Neurochemistry, 72, 2039-2046.

Gao, K., Sheehan, D. V., y Calabresse, J.R. (2009) Atypical antipsychotics in primary generalizad anxiety disorder or comorbid with mood disorders. Expert Review of Neurotherapeutics, 9, 1147-1158.

García-Portilla, M. P., Bascarán, M. T., Saiz, P. A., Mateos, M., González-Quirós, M., Pérez, P.,... Bobes, J. (2005). Effectiveness of venlafaxine in the treatment of alcohol dependence with comorbid depression. Actas Españolas de Psiquiatria, 33, 41-45.

García-Portilla, M. P., Bascarán, M. T., Sáiz, P. A., Parellada, M., Bousoño, y M., Bobes, J. (2011). Banco de instrumentos básicos para la práctica de la psquiatría clínica. $\sigma^{a}$ ed. Majadahonda: Comunicación y Ediciones Sanitarias, S.L.

Giner, J. (Coord). (2005). Consenso español sobre el trastorno de ansiedad generalizada. Sociedad Española de Psiquiatría y Sociedad Española de Psiquiatría Biológica. Barcelona: Ars Medica. 
Gonzalez, G., Severino, K., Sofuoglu, M., Poling, J., Olivito, A., Gonsai, K.,... Kosten, T. R. (2003. Tiagabine increases cocaine-free urines in cocaine-dependent methadone-treated patients: results of a randomized pilot study. Addiction, 98, 1625-1632.

González, G., Desai, R., Sofuoglu, M., Poling, J., Oliveto, A., Gonsai, K.,... Kosten, T. R. (2007). Clinical efficacy of gabapentin versus tiagabine for reducing cocaine use among cocaine dependent methadone-treated patients. Drug and Alcohol Dependence, 87, 1-9.

Goodman, W. K., Price, L. H., Rasmussen, S. A., Mazure, C., Fleischmann, R. L., Hill, C. L.,... Charney, D. S. (1989). The Yale-Brown Obsessive Compulsive Scale. I. Development, use, and reliability. Archives of General Psychiatry, 46, 1006-1011.

Goodwin, R. D., Lipsitz, J. D., Chapman, T. F., Mannuzza, S., Klein, D. F., y Fyer, A. J. (2006). Alcohol use disorders in relatives of patients with panic disorder. Compehensive Psychiatry, 47, 88-90.

Goossop, M. (1990). The development of a short opiate withdrawal scale (SOWS). Addictive Behaviors, 15, 487-490.

Gorman, J., Shear, K., Cowley, D., Cross, C. D., March, J., Roth, W.,... Wang, P.S. (2006). Guía clinica para el tratamiento del trastorno de angustia. En: Asociación Americana de Psiquiatría (Ed.) Guías clínicas para el tratamiento de los trastornos psiquiátricos. Compendio. (pp 827912). Barcelona: Ars Medica

Grant, B. F., Stinson, F. S., Dawson, D. A., Chou, S. P., Dufour, M. C., Compton, W.,... Kaplan, K. (2004). Prevalence and co-occurrence of substance use disorders and independent mood and anxiety disorders: results from the National Epidemiologic Survey on Alcohol and Related Conditions. Archives of General Psychiatry, 61, 807-816.

Hariri, A. R., y Weinberger, D. R. (2003). Functional neuroimaging of genetic variation in serotonergic neurotransmission. Genes, Brain, and Behavior, 2, 341-349.

Hasin, D. S., Trautman, K. D., Miele, G. M., Samet, S., Smith, M., y Endicott, J. (1996). Psychiatric Research Interview for Substance and Mental Disorders (PRISM): Reliability for substance abusers. American Journal of Psychiatry, 153, 1195-1201

Hasin, D. S., Stinson, F. S., Ogburn, E., y Grant, B. F. (2007). Prevalence, correlates, disability, and comorbidity of DSM-IV Alcohol Abuse and Dependence in the United States. Archives of General Psychiatry, 64, 830-842.

Hesse, M. (2009). Integrated psychological treatment for substance use and co-morbid anxiety or depression vs. treatment for substance use alone. A systematic review of the published literature. BMC Psychiatry, 9, 6.

Himmerich, H., Nickel, T., Dalal, M.A., y Müller, M. B. (2007). Gabapentin treatment in a female patient with panic disorder and adverse effects under carbamazepine during benzodiazepine withdrawal. Psychiatrische Praxis, 34, 93-94.
Hobbs, J. D. J., Kushner, M. G., Lee, S. S., Reardon, S. M., y Maurer, E. W. (2011). Meta-analysis of supplemental treatment for depressive and anxiety disorders in patients being treated for alcohol dependence. The American Journal on Addiction, 20, 319-329.

Hollander, E., y Dell'Osso, B. (2006). Topiramate plus paroxetine in treatment-resistant obsessive-compulsive disorder. International Clinical Psychopharmacology, 21, 189-191.

Jacobsen, L. K., Southwick, y S. M., Kosten, T. R. (2001). Substance use disorders in patients with posttraumatic stress disorder: a review of the literature. American Journal of Psychiatry, 158, 1184-1190.

Johnson, J. G., Cohen, P., Pine, D. S., Klein, D. F., Kasen, S., y Brook, J. S. (2000). Association between cigarette smoking and anxiety disordersduring adolescence and early adulthood. JAMA, 284, 2348-2351

Kampman, K. M., Volpicelli, J. R., McGinnis, D. E., Alterman, A. I., Weinrieb, R. M., D’Angelo, L.,... Epperson, L. E. (1998). Reliability and validity of the Cocaine Selective Severity Assessment. Addictive Behaviors, 23, 449-461.

Kampman, K. M., Pettinati, H., Lynch, K. G., Dackis, C., Sparkman, T., Weigley, C.,... O'Brien, C. P. (2004). A pilot trial of topiramate for the treatment of cocaine dependence. Drug and Alcohol Dependence, 75, 233-240.

Kaufman, J., Yang, B. Z., Douglas-Palumberi, H., Crouse-Artus, M., Lipschitz, D., Cristal, J. H.,... Gelenrter, J. (2007). Genetic and environmental predictors of early alcohol use. Biological Psychiatry, 61, 1228-1234.

Kessler, R. C., Chiu, W. T., Jin, R., Ruscio, A. M., Shear, K., y Walters, E. E. (2006). The epidemiology of panic attacks, panic disorder, and agoraphobia in the National Comorbidity Survey Replication. Archives of General Psychiatry, 63, 415-424.

Kinrys, G., Pollack, M. H., Simon, N. M., Worthington, J. J., Nardi, A. E., y Versiani, M. (2003). Valproic acid for the treatment of social anxiety disorder. International Clinical Psychopharmacology, 18, 169-172.

Koethe, D., Juelicher, A., Nolden, B. M., Braunwarth, W. D., Klosterkötter, J., Niklewski G,... Leweke, F. (2007). Oxcarbazepine-efficacy and tolerability during treatment of alcohol withdrawal: a double-blind, randomized, placebo-controlled multicenter pilot study. Alcoholism: Clinical and Experimental Research, 31, 1188-1194.

Koob, G., y Kreeb, M. J. (2007). Stress, dysregulation of drug reward pathways, and the transition to drug dependence. American Journal of Psychiatry, 164, 1149-1159.

Kranzler, M. R. (1996). Evaluation and treatment of anxiety symptoms and disorders in alcoholics. Journal of Clinical Psychiatry, 57, 15-21.

Kreeb, M. J., y Koob, G. F. (1998). Drug dependence: stress and dysregulation of brain reward pathways. Drug and Alcohol Dependence, 51, 23-47.

Kushner, M. G., Abrams, K., y Borchardt, C. (2000). The relationship between anxiety disorders and alcohol use 
disorders: a review of major perspectives and findings. Clinical Psychology Review, 20, 149-171.

Lanheim, A. S., Bakken, y K., Vaglum, P. (2006). Impact of comorbid psychiatric disorders on the outcome of substance abusers: a six year prospective follow-up in two Norwegian counties. BMC Psychiatry, 6, 44.

Laucht, M., Treutlein, J., Schmid, B., Blomeyer, D., Becker, K., Buchmann, A. F.,... Banaschewski, T. (2009). Impact of psychosocial adversity on alcohol intake in young adults: moderation by the LL genotype of the serotonin transporter polymorphism. Biological Psychiatry, 66, 102-109.

Leshner, A. I., y Koob, G. F. (1999). Drugs of abuse and the brain. Proceedings of the Association of American Physicians, 111, 99-108.

Liebowitz, M.R. (1987). Social phobia. Modern Problems of Pharmacopsychiatry, 22, 141-173.

Lindley, S. E., Carlson, E. B., y Hill, K. (2007). A randomized, double-blind, placebo-controlled trial of augmentation topiramate for chronic combat-related posttraumatic stress disorder. Journal of Clinical Psychopharmacology, 27, 677-681.

Llopis Llácer, J. J., y Castillo Aguilella, A. (2008). Efficacy of oxcarbazepine treatment in patients diagnosed with cocaine abuse/dependence. Adicciones, 20, 263-270.

Lydiard, R. B., Rickels, K., Herman, B., y Feltner, D. E. (2010). Comparative efficacy of pregabalin and benzodiazepines in treating the psychic and somatic symptoms of generalized anxiety disorder. The International Journal of Neuropsychopharmacology, 13, 229-241.

Madoz-Gúrpide, A., García Vicent, V., Luque Fuentes, E., y Ochoa Mangado, E. (2013). Variables predictivas del alta terapéutica entre pacientes con patología dual grave atendidos en una comunidad terapéutica de drogodependientes con unidad psiquiátrica. Adicciones, 25, 300-308.

Malek-Ahmadi, P., y Hanretta, A. T. (2004). Possible reduction in posttraumatic stress disorder symptoms with oxcarbazepine in a patient with bipolar disorder. The Annals of Pharmacotherapy, 38, 1852-1854.

Manzanares, J., García, M. S., Celorrio, M. L., Sánchez, M. M., y Rubio, G. (2010). Trastornos por ansiedad y adicciones. En: G. Haro, J. Bobes, M. Casas, J. Didia, G. Rubio (Eds.), Tratado sobre patología dual. Reintegrando la salud mental (pp. 531-571). Castellón: MRA Médica.

Marmorstein, N. R. (2012). Anxiety disorders and substance use disorders: different associations by anxiety disorder. Journal of Anxiety Disorders, 26, 88-94.

Marquenie, L. A., Schadé, A., van Balkom, A. J., Comijs, H. C., de Graaf, R., Vollebergh, W.,... van den Brink,, W. (2007). Origin of the comorbidity of anxiety disorders and alcohol dependence: findings of a general population study. European Addiction Research, 13, 39-49.

Martínez-Raga, J., Sabater, A., Perez-Galvez, B., Castellano, M., y Cervera, G. (2004). Add-on gabapentin in the treatment of opiate withdrawal. Progress in Neuropsychopharmacology and Biological Psychiatry, 28, 599-601.
Martinotti, G., Romanelli, R., Di Nicola, M., Reina, D., Mazza, M., y Janiri, L. (2007). Oxcarbazepine at high dosages for the treatment of alcohol dependence. The American Journal on Addictions, 16, 247-248.

Martinotti, G., Di Nicola, M., Tedeschi, D., Andreoli, S., Reina, D., Pomponi, M.,... Janiri, L. (2010a). Pregabalin versus naltrexone in alcohol dependence: a randomised, double-blind, comparison trial. Journal of Psychopharmacology, 24, 1367-1374.

Martinotti, G., di Nicola, M., Frustaci, A., Romanelli, R., Tedeschi, D., Guglielmo, R.,... Janiri, L. (2010b). Pregabalin, tiapride and lorazepam in alcohol withdrawal syndrome: a multi-centre, randomized, single-blind comparison trial. Addiction, 105, 288-299.

Mateo, Y., Lack, C. M., Morgan, D., Roberts, D. C., y Jones, S. R. (2005). Reduced dopamine terminal function and insensitivity to cocaine following cocaine binge self-administration and deprivation. Neuropsychopharmacology, 30, 1455-1463.

McKeehan, M. B., y Martin, D. (2002). Assessment and treatment of anxiety disorders and comorbid alcohol/ other drug dependency. Alcoholism Treatment Quarterly, 20, 45-59.

Merikangas, K. R., Mehta, R. L., Molnar, B. E., Walters, E. E., Swendsen, J. D., Aguilar-Gaziola, S.,... Kessler, R. C. (1998). Comorbidity of substance use disorders with mood and anxiety disorders: results of the International Consortium in Psychiatric Epidemiology. Addictive Behaviors, 23, 893-907.

Ministerio de Sanidad y Consumo. (2008) Guía de Práctica Clínica para el Manejo de Pacientes con Trastornos de Ansiedad en Atención Primaria. Agencia Laín Entralgo. Unidad de Evaluación de Tecnologías Sanitarias. Madrid

Montgomery, S. A., Herman, B. K., Schweizer, E., y Mandel, F. S. (2009). The efficacy of pregabalin and benzodiazepines in generalized anxiety disorder presenting with high levels of insomnia. International Clinical Psychopharmacology, 24, 214-222.

NICE. (2011) Generalised anxiety disorder and panic disorder (with or without agoraphobia) in adults. Management in primary, secondary and community care. National Clinical Guideline Number 113.

Nicolini, H., Herrera, K., Páez, F., Sánchez de Carmona, M., Orozco, B., Lodeiro, G.,... de la Fuenta, J.R. (1996). Traducción al español y confiabilidad de la Escala Yale-Brown para el trastorno obsesivo-compulsivo. Salud Mental, 5, 13-16.

North, C. S., Tivis, L., McMillen, J. C., Pfefferbaum, B., Spitznagel, E. L., Cox, J.,... Smith, E. M. (2002). Psychiatric disorders in rescue workers after the Oklahoma City bombing. American Journal of Psychiatry, 159, 857-859.

Nunes, E. V., McGrath, P. J., y Quitkin, F. M. (1995). Treating anxiety in patients with alcoholism. Journal of Clinical Psychiatry, 57, 3-9.

Ochoa, E., Salvador, E., Vicente, M., y Madoz, A. (2010). Comorbilidad psiquiátrica y adicciones. En: Pereiro 
Gómez, C. (Ed.), Manual de adicciones para médicos especialistas en formación (pp. 701-731). Madrid: DGPNSD

OECD (2012), OECD Health Data: Pharmaceutical market, OECD Health Statistics (database). doi: 10.1787/data00545-en (Recuperado el 30 de Enero de 2013)

Oslin, D. W., Berrettini, W., Kranzler, H. R., Pettinati, H., Gelernter, J., Volpicelli, J. R.,... O'Brien, C.P. (2003). A functional polymorphism of the mu-opioid receptor gene is associated with naltrexone response in alcohol-dependent patients. Neuropsychopharmacology, 28, 1546-1552.

Oulis, P., Florakis, A., Tzanoulinos, G., y Papadimitriou, G. N. (2008a). Pregabalin in the treatment of benzodiazepine-induced obsessive-compulsive disorder. Progress in Neuropsychopharmacology and Biological Psychiatry, 32, 2000-2001.

Oulis, P., Masdrakis, V. G., Karapoulios, E., Karakatsanis, N. A., Kouzoupis, A. V., Konstantakopoulos, G.,... Soldatos, C. R. (2008b) Pregabalin augmentation to sertraline-risperidone combination in the treatment of obsessive-compulsive disorder. Primary Care Companion to the Journal of Clinical Psychiatry, 10, 249.

Oulis, P., Masdrakis, V. G., Karapoulios, E., Karakatsanis, N. A., Kouzoupis, A. V., Konstantakopoulos, G.,... Soldatos, C. R. (2009). Tiagabine augmentation to fluvoxamine-risperidone combination in the treatment of obsessive-compulsive disorder. The World Journal of Biological Psychiatry, 10, 953-955.

Pacak, K., Tjurmina, O., Palkovits, M., Goldstein, D. S., Koch, C. A., Hoff, T.,... Chrousos, G.P. (2002). Chronic hypercortisolemia inhibits dopamine synthesis and turnover in the nucleus accumbens: an in vivo microdialysis study. Neuroendocrinology, 76, 148-157.

Pae, C. U., Marks, D. M., Han, C., Masand, P. S., y Patkar, A. A. (2009). Pregabalin augmentation of antidepressants in patients with accident-related posttraumatic stress disorder: an open label pilot study. International Clinical Psychopharmacology, 24, 29-33.

Pande, A. C., Davidson, J. R., Jefferson, J. W., Janney, C. A., Katzelnick, D. J., Weisler, R. H.,... Sutherland, S. M. (1999). Treatment of social phobia with gabapentin: a placebo-controlled study. Journal of Clinical Psychopharmacology, 19, 341-348.

Pande, A. C., Pollack, M. H., Crockatt, J., Greiner, M., Chouinard, G., Lydiard, R. B.,... Shiovitz, T. (2000). Placebo-controlled study of gabapentin treatment of panic disorder. Journal of Clinical Psychopharmacology, 20, 467-471.

Pande, A. C., Feltner, D. E., Jefferson, J. W., Davidson, J. R., Pollack, M., Stein, M. B.,... Werth, J. L. (2004). Efficacy of the novel anxiolytic pregabalin in social anxiety disorder: a placebo-controlled, multicenter study. Journal of Clinical Psychopharmacology, 24, 141-149.

Paparrigopoulos, T., Tzavellas, E., Karaiskos, D., Malitas, P., y Liappas, I. (2009). An open pilot study of tiagabine in alcohol dependence: tolerability and clinical effects. Journal of Psychopharmacology, 24, 1375-1380

Pasche, S. (2012). Exploring the Comorbisity of Anxiety and Substance Use Disorders. Current Psychiatry Reports, 14, 176-181

Patterson, W. M., Dohn, H. H., Bird, J., y Patterson, G. A. (1983). Evaluation of suicidal patients: the SAD PERSONS scale. Psychosomatics, 24, 343-345, 348-349.

Pettinati, H. M., Volpicelli, J. R., Kranzler, H. R., Luck, G., Rukstalis, M. R., y Cnaan, A. (2000). Sertraline treatment for alcohol dependence: interactive effects of medication and alcoholic subtype. Alcoholism: Clinical and Experimental Research, 24, 1041-1049.

Pezawas, L., Meyer-Lindenberg, A., Drabant, E. M., Verchinski, B. A., Munoz, K. E., Kolachana, B. S.,... Weinberger, D. R. (2005). 5-HTTLPR polymorphism impacts human cingulate-amygdala interactions: a genetic susceptibility mechanism for depression. Nature Neuroscience, 8, 828-834.

Piazza, P. V., Deminiere, J. M., le Moal, M., y Simon, H. (1990). Stress- and pharmacologically-induced behavioral sensitization increases vulnerability to acquisition of amphetamine self-administration. Brain Research, 514, 22-26.

Piazza, P. V., Maccari, S., Deminière, J. M., Le Moal, M., Mormède, P., y Simon, H. (1991). Corticosterone levels determine individual vulnerability to amphetamine self-administration. Proceedings of the National Academy of Sciences of the United States of America, 88, 2088-2092.

Pollack, M. H., Tiller, J., Xie, F., y Trivedi, M. H. (2008). Tiagabine in adult patients with generalized anxiety disorder: results from 3 randomized, double-blind, placebo-controlled, parallel-group studies. Journal of Clinical Psychopharmacology, 28, 308-316.

Posner, K., Brown, G. K., Stanley, B., Brent, D. A., Yershova, K. V., Oquendo, M. A.,... Mann, J. J. (2011). The Columbia-Suicide Severity Rating Scale: Initial Validity and Internal Consistency Findings From Three Multisite Studies With Adolescents and Adults. The American Journal of Psychiatry, 168, 1266-1277

Posternak, M. A., y Mueller, T. I. (2001). Assessing the risks and benefits of benzodiazepines for anxiety disorders in patients with a history of substance abuse or dependence. The American Journal on Addictions, 10, 48-68.

Radel, M., Vallejo, R. L., Iwata, N., Aragon, R., Long, J. C., Virkkunen, M.,... Goldman, D. (2005). Haplotype-based localization of an alcohol dependence gene to the 5 q34 \{gamma\}-aminobutyric acid type A gene cluster. Archives of General Psychiatry, 62, 47-55.

Randall, C., Thomas, S., y Thevos, A. (2001). Concurrent alcoholism and social anxiety disorder: a first step toward developing effective treatments. Alcoholism: Clinical and Experimental Research, 25, 210-220.

Reis, A. D., Castro, L. A., Faria, R., y Laranjeira, R. (2008). Craving decrease with topiramate in outpatient treat- 
ment for cocaine dependence: an open label trial. Revista Brasileira de Psiquiatria, 30, 132-135.

Regier, D. A., Farmer, M. E., Rae, D. S., Locke, B. Z., Keith, S. J., Judd, L. L.,... Goodwin, F. K. (1990). Comorbidity of mental disorders with alcohol and other drug abuse. Results from the Epidemiologic Catchment Area (ECA). JAMA, 264, 2511-2518.

Rosen, M. I., Pearsall, H. R., y Kosten, T. R. (1998). The effect of lamotrigine on naloxone-precipitated opiate withdrawal. Drug and Alcohol Dependence, 52, 173-176.

Rubio, G., Bermejo, J., Caballero, M. C., y Santo-Domingo, J. (1998). Validación de la prueba para la identificación de trastornos por uso de alcohol (AUDIT) en atención primaria. Revista Clinica Española, 198, 11-14.

Rubio, G., López-Muñoz, F., y Álamo, C. (2002). Tratamiento farmacológico de los trastornos de ansiedad en dependientes de sustancias. En: G. Rubio, F. López-Muñoz, C. Álamo, J. Santo-Domingo (Eds.), Trastornos psiquiátricos y abuso de sustancias (pp. 315-329) Madrid: Editorial Médica Panamericana

Rubio, G., López-Muñoz, y F., Alamo, C. (2006). Effects of lamotrigine in patients with bipolar disorder and alcohol dependence. Bipolar Disorders, 8, 289-293.

Rubio, G., Martínez-Gras, I., y Manzanares, J. (2009). Modulation of impulsivity by topiramate: implications for the treatment of alcohol dependence. Journal of Clinical Psychopharmacology, 29, 584-589.

Sáiz, P. A., García-Portilla, M. P., y Bobes, J. (2011). Instrumentos de evaluación. En: J. Bobes, J. Giner, J. Sáiz (Coord.), Suicidio y psiquiatría: Recomendaciones preventivas y manejo del comportamiento suicida (pp. 95-122) Madrid: Editorial Triacastela

San Molina, L. (Coord.) (2004). Consenso sobre patología dual. Sociedad Española de Psiquiatría y Sociedad Española de Psiquiatría Biológica. Barcelona: Ars Medica.

San Molina, L., Casas, M., Arranz, B., Bruguera, E., Busquets, E., Camps, N.,... Valls Lapica, J. J. (2005). Patología dual. En: P. A. Soler, J. Gascón (Coord.), Recomendaciones terapéuticas en los trastornos mentales RTM-III (pp. 63-82) Barcelona: Ars Medica

Sareen, J., Chartier, M., Paulus, M. P., y Stein, M. B. (2006). Illicit drug use and anxiety disorders: findings from two community surveys. Psychiatry Research, 142, 11-17.

Sattar, S. P., Schultz, S. K., Arndt, S., Soundy, T., y Petty, F. (2007). Long-term adjunctive quetiapine may reduce substance use-a preliminary retrospective study. South Dakota Medicine, 60, 437-443.

Saunders, J. B., Aasland, O. G., Babor, T. F., de la Fuente, J. R., y Grant, M. (1993). Development of the Alcohol Use Disorders Identification Test (AUDIT): WHO Collaborative Project on Early Detection of Persons with Harmful Alcohol Consumption-II. Addiction, 88, 791-804.

Schadé, A., Marquenie, L. A., van Balkom, A. J., Koeter, M. W., de Beurs, E., van den Brink, W.,... van Dyck, R.
(2005). The effectiveness of anxiety treatment on alcohol-dependent patients with a comorbid phobic disorder: a randomized controlled trial. Alcoholism: Clinical and Experimental Research, 29, 794-800.

Schäfer, I., y Najavits, L. M. (2007). Clinical challenges in the treatment of patients with posttraumatic stress disorder and substance abuse. Curr Opin Psychiatry, 20, 614-618.

Schneier, F. R., Foose, T. E., Hasin, D. S., Heimberg, R. G., Liu, S. M., Grant, B. F.,... Blanco, C. (2010). Social anxiety disorder and alcohol use disorder co-morbidity in the National Epidemiologic Survey on Alcohol and Related Conditions. Psychological Medicine, 40, 977-988.

Schultz, W., Dayan, P., y Montague, P. R. (1997). A neural substrate of prediction and reward. Science, 275, 1593-1599.

Secades, R., Rodriguez-García, E., Valderrey, J., Fernández-Hermida, J., Vallejo, G., y Jiménez-García, J. (2003). El consumo de psicofármacos en pacientes que acuden a Atención Primaria en el Principado de Asturias (España). Psicothema, 15, 650-655.

Sheehan, D. V., Lecrubier, Y., Harnett-Sheehan, K., Janavs, J., Weiller, E., Kreskiner, A.,... Dunbar, G. (1997). Reliability and validity of the MINI Internacional Neuropsychiatric Interview (MINI): according to the SCID-P and its reliability. European Psychiatry, 12, 232-41.

Sheehan, D. V., Sheehan, K. H., Raj, B. A., y Janavs, J. (2007). An open-label study of tiagabine in panic disorder. Psychopharmacology Bulletin, 40, 32-40.

Sociedad Española de Patología Dual. (2009). Estudio epidemiológico sobre la prevalencia de patología dual en la Comunidad de Madrid. Recuperado de http://www.patologiadual.es / docs /estudio_epidemiologico_PD_CE2008_NOV08.pdf

Spila, B., Szumiłło, A. (2006). Gabapentin (GBP) in panic disorders-case report. Psychiatria Polska, 40, 1061-1068.

Stein, M. B., Kerridge, C., Dimsdale, J. E., y Hoyt, D. B. (2007). Pharmacotherapy to prevent PTSD: Results from a randomized controlled proof-of-concept trial in physically injured patients. Journal of Traumatic Stress, 20, 923-932.

Stein, D. J., Baldwin, D. S., Baldinetti, F., y Mandel, F. (2008). Efficacy of pregabalin in depressive symptoms associated with generalized anxiety disorder: a pooled analysis of 6 studies. European Neuropsychopharmacology, 18, 422-430.

Stein, D. J., Bruce Lydiard, R., Herman, B. K., y Mandel, F. S. (2009). Impact of gastrointestinal symptoms on response to pregabalin in generalized anxiety disorder: results of a six-study combined analysis. International Clinical Psychopharmacology, 24, 126-132.

Sullivan, J. T., Sykora, K., Schneiderman, J., Naranjo, C. A., y Sellers, E. M. (1989). Assessment of alcohol withdrawal: the revised clinical institute withdrawal assessment for alcohol scale (CIWA-Ar). British Journal of Addiction, 84, 1353-1357.

Tejero, A., Trujols, J., y Siñol, N. (2003a). Evaluación del craving de cocaína. En: A. Tejero, J. Trujols (Eds.), In- 
strumentos clínicos para la evaluación de la dependencia de cocaina (pp. 23-50). Barcelona: Ars Medica.

Tejero, A., Trujols, J., y Gil, L. (2003b). Evaluación del síndrome de abstinencia de cocaína. En: A. Tejero, J. Trujols (Eds.), Instrumentos clínicos para la evaluación de la dependencia de cocaina (pp13-22). Barcelona: Ars Medica.

Terra, M. B., Barros, H. M. T., Stein, A. T., Figueira, I., Jorge, M. R., Palermo, L. H.,... Da Silveira, D. X. (2006). Social anxiety disorder in 300 patients hospitalizad for alcoholism in Brazil: high prevalence and undertreatment. Compehensive Psychiatry, 47, 463-467.

Thomas, S. E., Randall, P. K., Book, S. W., y Randall, C. L. (2008). A complex relationship between co-ocurring social anxiety and alcohol use disorders: what effect does treating anxiety have on drinking? Alcoholism: Clinical and Experimental Research, 32, 77-84

Tiet, Q. Q., y Mausbach, B. (2007). Treatments for patients with dual diagnosis: a review. Alcoholism: Clinical and Experimental Research, 31, 513-536.

Tiffany, S. T., Singleton, E., Haertzen, C. A., y Henningfield, J.E. (1993). The development of a cocaine craving questionnaire. Drug and Alcohol Dependence, 34, 19-28.

Torrens, M., Serrano, D., Astals, M., Pérez-Domínguez, G., y Martín-Santos, R. (2004). Diagnosing comorbid psychiatric disorders in substance abusers: validity of the Spanish versions of the Psychiatric Research Interview for Substance and Mental Disorders and the Structured Clinical Interview for DSM-IV. American Journal of Psychiatry, 161, 1231-1237.

Tucker, P., Trautman, R. P., Wyatt, D. B., Thompson, J., Wu, S. C., Capece, J. A.,... Rosenthal, N. R. (2007). Efficacy and safety of topiramate monotherapy in civilian posttraumatic stress disorder: a randomized, double-blind, placebo-controlled study. Journal of Clinical Psychiatry, 68, 201-206.

Tyrer, P., Murphy, S., y Riley, P. (1990). The benzodiazepine withdrawal symptom questionnaire. Journal of Affective Disorders, 19, 53-61.

Uhart, M., McCaul, M. E., Oswald, L. M., Choi, L., y Wand, G. S. (2004). GABRA6 gene polymorphism and an attenuated stress response. Molecular Psychiatry, 9, 998-1006.

Uhart, M., y Wand, G. S. (2009). Stress, alcohol and drug interaction: an update of human research. Addiction Biology, 14, 43-64.

Ursano, R. J., Bell, C., Eth, S., Friedman, M., Norwood, A., Pfefferbaum, B.,... Benedek, D. M. (2006). Guía clínica para el tratamiento de los trastornos por estrés agudo y estrés postraumático. En: Guías clínicas para el tratamiento de los trastornos psiquiátricos. Compendio 2006. Asociación Americana de Psiquiatría (pp. 1133-1266). Barcelona: Ars Medica.

Uzun, O. (2010). Lamotrigine as an augmentation agent in treatment-resistant obsessive-compulsive disorder: a case report. Journal of Psychopharmacology, 24, 425-427.
Van Ameringen, M., Manzini, C., Pipe, B., Oakman, J., y Bennett, M. (2004). An open trial of topiramate in the treatment of generalized social phobia. Journal of Clinical Psychiatry, 65, 1674-1678.

Van Ameringen, M., Mancini, C., Patterson, B., y Bennett, M. (2006). Topiramate augmentation in treatment-resistant obsessive-compulsive disorder: a retrospective, open-label case series. Depression and Anxiety, 23, 1-5.

Van Laar, M., van Dorsselaer, S., Monshouwer, K., y de Graaf, R. (2007). Does cannabis use predict the first incidence of mood and anxiety disorders in the adult population? Addiction, 102, 1251-1260.

Verduin, M. L., McKay, S., y Brady, K. T. (2007). Gabapentin in comorbid anxiety and substance use. The American Journal on Addictions, 16, 142-143.

Volkow, N. D., Fowler, J. S., y Wang, G. J. (2003). Positron emisión tomography and single-photon emision computed tomography in substance abuse research. Seminars in Nuclear Medicine, 33, 114-128.

Wand, G. S., Oswald, L. M., McCaul, M. E., Wong, D. F., Johnson, E., Zhou, Y.,... Kumar, A. (2007). Association of amphetamine-induced striatal dopamine release and cortisol responses to psychological stress. Neuropsychopharmacology, 32, 2310-2320.

Watkins, K. E., Hunter, S. B., Burnam, M. A., Pincus, H. A., y Nicholson, G. (2005). Review of treatment recommendations for persons with a co-occurring affective or anxiety and substance use disorder. Psychiatric Services, 56, 913-926.

Weiss, F., Parsons, L. H., Schulteis, G., Hyytiä, P., Lorang, M. T., Bloom, F. E.,... Koob, G. F. (1996). Ethanol self-administration restores withdrawal-associated deficiencies in accumbal dopamine and 5-hydroxytryptamine release in dependent rats. The Journal of Neuroscience, 16, 3474-3485.

Winhusen, T. M., Somoza, E. C., Harrer, J. M., Mezinskis, J. P., Montgomery, M. A., Goldsmith, R. J.,... Elkashef, A. (2005). A placebo-controlled screening trial of tiagabine, sertraline and donepezil as cocaine dependence treatments. Addiction, 100, 68-77.

Winhusen, T., Somoza, E., Ciraulo, D. A., Harrer, J. M., Goldsmith, R. J., Grabowski, J.,... Elkashef, A. (2007). A double-blind, placebo-controlled trial of tiagabine for the treatment of cocaine dependence. Drug and Alcohol Dependence, 91, 141-148.

Wüst, S., Van Rossum, E. F., Federenko, I. S., Koper, J. W., Kumsta, R., y Hellhammer, D. H. (2004). Common polymorphisms in the glucocorticoid receptor gene are associated with adrenocortical responses to psychosocial stress. The Journal of Clinical Endocrinology $\mathcal{E}$ Metabolism, 89, 565-573.

Zullino, D. F., Cottier, A. C., y Besson, J. (2002) Topiramate in opiate withdrawal. Progress in Neuropsychopharmacology and Biological Psychiatry, 26, 1221-1223. 
Zvolensky, M.J., Bernstein, A., Sachs-Ericsson, N., Schmidt, N. B., Buckner, J. D., y Bonn-Miller, M. O. (2006). Lifetime associations between cannabis, use, abuse, and dependence and panic attacks in a representative sample. Journal of Psychiatry Research, 40, 477-486.

Zwanzger, P., Eser, D., Nothdurfter, C., Baghai, T. C., Möller, H. J., Padberg, F., y Rupprecht, R. (2009). Effects of the GABA-reuptake inhibitor tiagabine on panic and anxiety in patients with panic disorder. Pharmacopsychiatry, 42, 266-269. 